See discussions, stats, and author profiles for this publication at: https://www.researchgate.net/publication/252063492

\title{
Asymptotics and Power Allocation for State Estimation Over Fading Channels
}

Article in IEEE Transactions on Aerospace and Electronic Systems · January 2011

DOI: 10.1109/TAES.2011.5705695

CITATIONS

19

3 authors, including:

Subhrakanti Dey

University of Melbourne

161 PUBLICATIONS 1,971 CITATIONS

SEE PROFILE
READS

57

Jamie Scott Evans
University of Melbourne
243 PUBLICATIONS 3,902 CITATIONS
SEE PROFILE 


\section{Asymptotics and Power Allocation for State Estimation Over Fading Channels}

\author{
ALEX S. LEONG \\ SUBHRAKANTI DEY, Senior Member, IEEE \\ JAMIE S. EVANS \\ University of Melborne
}

State estimation of linear systems using analog amplify and forwarding with multiple sensors, for both multiple access and orthogonal access schemes is considered. Optimal state estimation can be achieved at the fusion center using a time-varying Kalman filter. We show that in many situations, the estimation error covariance decays at a rate of $1 / M$ when the number of sensors $M$ is large. We consider optimal allocation of transmission powers that 1) minimizes the sum power usage subject to an error covariance constraint, and 2) minimizes the error covariance subject to a sum power constraint. In the case of fading channels with channel-state information, the optimization problems are solved using a greedy approach, while for fading channels without channel state information (CSI) but with channel statistics available, a suboptimal linear estimator is derived.

Manuscript received April 21, 2008; revised June 9, 2009; released for publication October 20, 2009.

IEEE Log No. T-AES/47/1/940051.

Refereeing of this contribution was handled by T. Luginbuhl.

This work was supported by the Australian Research Council.

Authors' address: Dept. of Electrical and Electronic Engineering, University of Melbourne, Grattan Street, Parkville, Melbourne, Victoria 3010, Australia, E-mail: (sdey@unimelb.edu.au).

0018-9251/11/\$26.00 (c) 2011 IEEE

\section{INTRODUCTION}

Wireless sensor networks are collections of sensors which can communicate with each other or to a central node or base station through wireless links. Potential uses include environment and infrastructure monitoring, healthcare and military applications, to name a few. Often these sensors will have limited energy and computational ability, which imposes severe constraints on system design, and signal processing algorithms that can efficiently utilise these resources have attracted great interest.

In recent years there has been considerable literature on estimation and detection schemes designed specifically for use in wireless sensor networks. Work on detection in wireless sensor networks includes [1], which studies the asymptotic optimality of using identical sensors in the presence of energy constraints, and [2]-[4] which derive fusion rules for distributed detection in the presence of fading. Parameter estimation or estimation of constant signals is studied in, e.g., [5]-[8], where issues of quantization and optimization of power usage are addressed. Type-based methods for detection and estimation of discrete sources are proposed and analyzed in [9]-[11]. Estimation of fields has been considered in, e.g., [12]-[14].

A promising scheme for distributed estimation in sensor networks is analog amplify and forward [15] (in distributed detection, analog forwarding has also been considered in, e.g., [16], [17]), where measurements from the sensors are transmitted directly (possibly scaled) to the fusion center without any coding, which is motivated by optimality results on uncoded transmissions in point-to-point links $[18,19]$. (Other related information-theoretic results include [20], [21].) Analog forwarding schemes are attractive due to their simplicity as well as the possibility of real-time processing since there is no coding delay. In [15] the asymptotic (large number of sensors) optimality of analog forwarding for estimating an independent and identically distributed (IID) scalar Gaussian process was shown, and exact optimality was later proved for a "symmetric" sensor network [22]. Analog forwarding with optimal power allocation is studied in [23] and [24] for multi-access and orthogonal schemes, respectively. Lower bounds and asymptotic optimality results for estimating independent vector processes is addressed in [25]. Estimation with correlated data between sensors is studied in [26], [27]. Other aspects of the analog forwading technique that have been studied include the use of different network topologies [28], other multiple access schemes such as slotted ALOHA [29], and consideration of the impact of channel estimation errors [30] on estimation performance.

Most of the previous work on analog forwarding has dealt with estimation of processes which are 
either constant or IID over time. In this paper we address the estimation of dynamical systems using analog forwarding of measurements. In particular, we consider the problem of state estimation of discrete-time linear systems using multiple sensors. As is well known, optimal state estimation of a linear system can be achieved using a Kalman filter. Other work on Kalman filtering in sensor networks includes studies of optimal sensor data quantization [31], Kalman filtering using one-bit quantized observations [32] where performance is shown to lie within a constant factor of the standard Kalman filter, and estimation of random fields with reduced-order Kalman filters [14]. Another related area with a rich history is that of distributed Kalman filtering, where the main objectives include doing local processing at the individual sensor level to reduce the computations required at the fusion center $[33,34]$ or forming estimates at each of the individual sensors in a completely decentralized fashion without any fusion center [35]. However in our work we assume that computational resources available at the sensors are limited so that they will only take measurements and then transmit them to the fusion center for further processing, using uncoded analog forwarding.

In this paper we mainly focus on estimation of scalar linear dynamical systems ${ }^{1}$ using multiple sensors, as the vector case introduces additional difficulties such that only partial results can be obtained. We are interested in deriving the asymptotic behaviour of the error covariance with respect to the number of sensors for these schemes, as well as optimal transmission power allocation to the sensors under a constraint on the error covariance at the fusion center or a sum power constraint at the sensor transmitters. We consider both static and fading channels, and in the context of fading channels, we consider various levels of availability of channel state information (CSI) at the transmitters and the fusion center. More specifically, we make the following key contributions.

1) We show that (for static channels with full CSI) for the multi-access scheme, the asymptotic estimation error covariance can be driven to the process noise covariance (which is the minimum attainable error) as the number of sensors $M$ goes to infinity, even when the transmitted signals from each sensor are scaled by $1 / \sqrt{M}$ (which implies that total transmission power across all sensors remains bounded while each sensor's transmission power goes to zero). This is a particularly attractive result since sensor networks operate in an energy-limited environment. For the orthogonal access scheme, this result holds when the transmitted signals are unscaled, but does not hold when the transmitted signals are scaled by $1 / \sqrt{M}$.

\footnotetext{
${ }^{1}$ By scalar linear system we mean that both the states and individual sensor measurements are scalar.
}

2) The convergence rate of these asymptotic results (when they hold) is shown to be $1 / M$ although it is seen via simulation results that the asymptotic approximations are quite accurate even for $M=20$ to 30 sensors.

3) In the case of a small to moderate number of sensors, we derive a comprehensive set of optimal sensor transmit power allocation schemes for multi-access and orthogonal medium access schemes over both static and fading channels. For static channels, we minimize total transmission power at the sensors subject to a constraint on the steady-state Kalman estimation error covariance, and also solve a corresponding converse problem: minimizing steady-state error covariance subject to a sum power constraint at the sensor transmitters. For fading channels (with full CSI), we solve similar optimization problems, except that the error covariance (either in the objective function or the constraint) is considered at a per-time-instant basis since there is no well-defined steady-state error covariance in this case. For the fading channel case with no CSI (either amplitude or phase), the results are derived for the best linear estimator, which relies on channel statistics information and can be applied to non-zero mean fading channels. It is shown that these optimization problems can be posed as convex optimization problems. Moreover, the optimization problems will turn out to be very similar to problems previously studied in the literature (albeit in the context of distributed estimation of a static random source), namely, [23, 24], and can actually be solved in closed form.

4) Numerical results demonstrate that for static channels, optimal power allocation results in more benefit for the orthogonal medium access scheme compared with the multi-access scheme, whereas for fading channels, it is seen that having full CSI is clearly beneficial for both schemes although the performance improvement via the optimal power allocation scheme is more substantial for the orthogonal scheme than for the multi-access scheme.

The rest of the paper is organized as follows. Section II specifies our scalar models and preliminaries, and gives a number of examples between multi-access and orthogonal access schemes, which show that in general one scheme does not always perform better than the other. We investigate the asymptotic behaviour for a large number of sensors $M$ in Section III. Power allocation is considered in Section IV, where we formulate and solve optimization problems for 1) an error covariance constraint, and 2) a sum power constraint. We first do this for static channels, before focusing on fading channels. In the case where we have CSI we use a greedy approach by performing the optimization at each time step. When we don't have CSI, we 
derive a suboptimal linear estimator similar to [36]-[38], which can be used for non-zero mean fading. Numerical studies are presented in Section V. Extensions of our model to vector and multiple-input, multiple-output (MIMO) systems is considered in Section VI, where we formulate the models and optimization problems and outline some of the difficulties involved.

\section{MODELS AND PRELIMINARIES}

Throughout this paper, $i$ represents the sensor index and $k$ represents the time index. Let the scalar linear system be

$$
x_{k+1}=a x_{k}+w_{k}
$$

with the $M$ sensors each observing

$$
y_{i, k}=c_{i} x_{k}+v_{i, k}, \quad i=1, \ldots, M
$$

with $w_{k}$ and $v_{i, k}$ being zero-mean Gaussians having variances $\sigma_{w}^{2}$ and $\sigma_{i}^{2}$, respectively, with the $v_{i, k} \mathrm{~s}$ being independent between sensors. Note that the sensors can have different observation matrices $c_{i}$ and measurement noise variances $\sigma_{i}^{2}$, and we allow $a$ and $c_{i}$ to take on both positive and negative values. It is assumed that the parameters $a, c_{i}, \sigma_{w}^{2}$ and $\sigma_{i}^{2}$ are known. ${ }^{2}$ Furthermore, we assume that the system is stable, i.e., $|a|<1$.

\section{A. Multi-Access Scheme}

In the (nonorthogonal) multi-access scheme, the fusion center receives the sum

$$
\tilde{z}_{k}=\sum_{i=1}^{M} \tilde{\alpha}_{i, k} \tilde{h}_{i, k} y_{i, k}+\tilde{n}_{k}
$$

where $\tilde{n}_{k}$ is zero-mean complex Gaussian with variance $2 \sigma_{n}^{2}, \tilde{h}_{i, k}$ are the complex-valued channel gains, and $\tilde{\alpha}_{i, k}$ are the complex-valued multiplicative amplification factors in an amplify and forward scheme. We assume that all transmitters have access to their complex CSI, ${ }^{3}$ and the amplification factors have the form

$$
\tilde{\alpha}_{i, k}=\alpha_{i, k} \frac{\tilde{h}_{i, k}^{*}}{\left|\tilde{h}_{i, k}\right|}
$$

where $\alpha_{i, k}$ is real-valued, i.e., we assume distributed transmitter beamforming. Defining $h_{i, k} \equiv\left|\tilde{h}_{i, k}\right|, z_{k} \equiv$ $\Re\left[\tilde{z}_{k}\right], n_{k} \equiv \Re\left[\tilde{n}_{k}\right]$, we then have

$$
z_{k}=\sum_{i=1}^{M} \alpha_{i, k} h_{i, k} y_{i, k}+n_{k} \text {. }
$$

\footnotetext{
${ }^{2}$ We assume that these parameters are static or very slowly time varying, and hence can be accurately determined beforehand using appropriate parameter estimation/system identification algorithms.

${ }^{3}$ The case where the channel gains are unknown but channel statistics are available is addressed in Section IVE. This can also be used to model the situation where perfect phase synchronization cannot be achieved [25].
}

Note that the assumption of CSI at the transmitters is important in order for the signals to add up coherently in (2). In principle, it can be achieved by the distributed synchronization schemes described in, e.g., [39], [40], but may not be feasible for large sensor networks. However, in studies such as [16], [39], it has been shown in slightly different contexts that for moderate amounts of phase error, much of the potential performance gains can still be achieved.

Continuing further, we may write

$$
z_{k}=\sum_{i=1}^{M} \alpha_{i, k} h_{i, k} c_{i} x_{k}+\sum_{i=1}^{M} \alpha_{i, k} h_{i, k} v_{i, k}+n_{k}=\bar{c}_{k} x_{k}+\bar{v}_{k}
$$

where $\bar{c}_{k} \equiv \sum_{i=1}^{M} \alpha_{i, k} h_{i, k} c_{i}$ and $\bar{v}_{k} \equiv \sum_{i=1}^{M} \alpha_{i, k} h_{i, k} v_{i, k}+n_{k}$. Hence we have the following linear system:

$$
x_{k+1}=a x_{k}+w_{k}, \quad z_{k}=\bar{c}_{k} x_{k}+\bar{v}_{k}
$$

with $\bar{v}_{k}$ having variance $\bar{r}_{k} \equiv \sum_{i=1}^{M} \alpha_{i, k}^{2} h_{i, k}^{2} \sigma_{i}^{2}+\sigma_{n}^{2}$. Define the state estimate and error covariance as

$$
\begin{aligned}
\hat{x}_{k+1 \mid k} & =\mathbb{E}\left[x_{k+1} \mid\left\{z_{0}, \ldots, z_{k}\right\}\right] \\
P_{k+1 \mid k} & =\mathbb{E}\left[\left(x_{k+1}-\hat{x}_{k+1 \mid k}\right)^{2} \mid\left\{z_{0}, \ldots, z_{k}\right\}\right]
\end{aligned}
$$

where again $P_{k+1 \mid k}$ is scalar. Then it is well known that optimal estimation of the state $x_{k}$ in the minimum mean squared error (MMSE) sense can be achieved using a (in general time-varying) Kalman filter [41]. Using the shorthand notation $P_{k+1}=P_{k+1 \mid k}$, the error covariance satisfies the recursion

$$
P_{k+1}=a^{2} P_{k}-\frac{a^{2} P_{k}^{2} \bar{c}_{k}^{2}}{\bar{c}_{k}^{2} P_{k}+\bar{r}_{k}}+\sigma_{w}^{2}=\frac{a^{2} P_{k} \bar{r}_{k}}{\bar{c}_{k}^{2} P_{k}+\bar{r}_{k}}+\sigma_{w}^{2} .
$$

We also remark that even if the noises are non-Gaussian, the Kalman filter is still the best linear estimator.

\section{B. Orthogonal Access Scheme}

In the orthogonal access scheme each sensor transmits its measurement to the fusion center via orthogonal channels (e.g., using FDMA or CDMA), so that the fusion center receives

$$
\tilde{z}_{i, k}=\tilde{\alpha}_{i, k} \tilde{h}_{i, k} y_{i, k}+\tilde{n}_{i, k}, \quad i=1, \ldots, M
$$

with the $\tilde{n}_{i, k}$ s being independent, zero-mean complex Gaussian with variance $2 \sigma_{n}^{2}, \forall i$. We again assume CSI at the transmitters and use $\tilde{\alpha}_{i, k}=\alpha_{i, k} \tilde{h}_{i, k}^{*} /\left|\tilde{h}_{i, k}\right|$ with $\alpha_{i, k} \in \mathbb{R}$. Let $h_{i, k} \equiv\left|\tilde{h}_{i, k}\right|, z_{i, k} \equiv \Re\left[\tilde{z}_{i, k}\right], n_{i, k} \equiv \Re\left[\tilde{n}_{i, k}\right]$. The situation is then equivalent to the linear system (using the superscript " $o$ " to distinguish some quantities in the orthogonal scheme from the multi-access scheme):

$$
x_{k+1}=a x_{k}+w_{k}, \quad \mathbf{z}_{k}^{o}=\overline{\mathbf{C}}_{k}^{o} x_{k}+\overline{\mathbf{v}}_{k}^{o}
$$


where

$$
\begin{aligned}
\mathbf{z}_{k}^{o} \equiv\left[\begin{array}{c}
z_{1, k} \\
\vdots \\
z_{M, k}
\end{array}\right] \\
\overline{\mathbf{C}}_{k}^{o} \equiv\left[\begin{array}{c}
\alpha_{1, k} h_{1, k} c_{1} \\
\vdots \\
\alpha_{M, k} h_{M, k} c_{M}
\end{array}\right] \\
\overline{\mathbf{v}}_{k}^{o} \equiv\left[\begin{array}{c}
\alpha_{1, k} h_{1, k} v_{1, k}+n_{1, k} \\
\vdots \\
\alpha_{M, k} h_{M, k} v_{M, k}+n_{M, k}
\end{array}\right]
\end{aligned}
$$

with the covariance of $\overline{\mathbf{v}}_{k}^{o}$ being it is well known that if $\left\{x_{k}\right\}$ is stationary, we have $\mathbb{E}\left[x_{k}^{2}\right]=\sigma_{w}^{2} /\left(1-a^{2}\right), \forall k$. In both the multi-access and orthogonal schemes, the transmit powers are then

$$
\gamma_{i, k}=\alpha_{i, k}^{2}\left(c_{i}^{2} \frac{\sigma_{w}^{2}}{1-a^{2}}+\sigma_{i}^{2}\right) \text {. }
$$

\section{Steady-State Error Covariance}

In this and the next few sections, we let $\tilde{h}_{i, k}=\tilde{h}_{i}$ (and hence $h_{i, k}=h_{i}$ ), $\forall k$ be time invariant, deferring the discussion of time-varying channels until Section IVD. We also assume in this case that $\alpha_{i, k}=$ $\alpha_{i}, \forall k$, i.e., the amplification factors don't vary with time, and we drop the subscript $k$ from quantities such as $\bar{c}_{k}$ and $\bar{r}_{k}$.

$$
\overline{\mathbf{R}}_{k}^{o} \equiv\left[\begin{array}{cccc}
\alpha_{1, k}^{2} h_{1, k}^{2} \sigma_{1}^{2}+\sigma_{n}^{2} & 0 & \cdots & 0 \\
0 & \alpha_{2, k}^{2} h_{2, k}^{2} \sigma_{2}^{2}+\sigma_{n}^{2} & \cdots & 0 \\
\vdots & \vdots & \ddots & \vdots \\
0 & 0 & \cdots & \alpha_{M, k}^{2} h_{M, k}^{2} \sigma_{M}^{2}+\sigma_{n}^{2}
\end{array}\right] .
$$

The state estimate and error covariance are now defined as

$$
\begin{aligned}
\hat{x}_{k+1 \mid k}^{o} & =\mathbb{E}\left[x_{k+1} \mid\left\{\mathbf{z}_{0}^{o}, \ldots, \mathbf{z}_{k}^{o}\right\}\right] \\
P_{k+1 \mid k}^{o} & =\mathbb{E}\left[\left(x_{k+1}-\hat{x}_{k+1 \mid k}^{o}\right)^{2} \mid\left\{\mathbf{z}_{0}^{o}, \ldots, \mathbf{z}_{k}^{o}\right\}\right] .
\end{aligned}
$$

Optimal estimation of $x_{k}$ in the orthogonal access scheme can also be achieved using a Kalman filter, with the error covariance now satisfying the recursion

$$
P_{k+1}^{o}=a^{2} P_{k}^{o}-a^{2}\left(P_{k}^{o}\right)^{2} \overline{\mathbf{C}}_{k}^{o T}\left(\overline{\mathbf{C}}_{k}^{o} P_{k}^{o} \overline{\mathbf{C}}_{k}^{o T}+\overline{\mathbf{R}}_{k}^{o}\right)^{-1} \overline{\mathbf{C}}_{k}^{o}+\sigma_{w}^{2}
$$

where $\overline{\mathbf{C}}_{k}^{o}$ and $\overline{\mathbf{R}}_{k}^{o}$ as defined above are, respectively, a vector and a matrix. To simplify the expressions, note that

$$
\overline{\mathbf{C}}_{k}^{o T}\left(\overline{\mathbf{C}}_{k}^{o} P_{k}^{o} \overline{\mathbf{C}}_{k}^{o T}+\overline{\mathbf{R}}_{k}^{o}\right)^{-1} \overline{\mathbf{C}}_{k}^{o}=\frac{\overline{\mathbf{C}}_{k}^{o T} \overline{\mathbf{R}}_{k}^{o^{-1}} \overline{\mathbf{C}}_{k}^{o}}{1+P_{k}^{o} \overline{\mathbf{C}}_{k}^{o T} \overline{\mathbf{R}}_{k}^{o-1} \overline{\mathbf{C}}_{k}^{o}}
$$

which can be shown using the matrix inversion lemma. Hence

$$
P_{k+1}^{o}=\frac{a^{2} P_{k}^{o}}{1+P_{k}^{o} \overline{\mathbf{C}}_{k}^{o^{T}} \overline{\mathbf{R}}_{k}^{o^{-1}} \overline{\mathbf{C}}_{k}^{o}}+\sigma_{w}^{2}
$$

where one can also easily compute $\overline{\mathbf{C}}_{k}^{o T} \overline{\mathbf{R}}_{k}^{o^{-1}} \overline{\mathbf{C}}_{k}^{o}=$ $\sum_{i=1}^{M} \alpha_{i, k}^{2} h_{i, k}^{2} c_{i}^{2} /\left(\alpha_{i, k}^{2} h_{i, k}^{2} \sigma_{i}^{2}+\sigma_{n}^{2}\right)$. The advantage of the orthogonal scheme is that we do not need carrier-level synchronization among all sensors, but only require synchronization between each individual sensor and the fusion center [24].

\section{Transmit Powers}

The power $\gamma_{i, k}$ used at time $k$ by the $i$ th sensor in transmitting its measurement to the fusion center is defined as $\gamma_{i, k}=\alpha_{i, k}^{2} \mathbb{E}\left[y_{i, k}^{2}\right]$. For stable scalar systems,
From Kalman filtering theory, we know that the steady-state (as $k \rightarrow \infty$ ) error covariance $P_{\infty}$ (provided it exists) in the multi-access scheme satisfies (cf. (4))

$$
P_{\infty}=\frac{a^{2} P_{\infty} \bar{r}}{\bar{c}^{2} P_{\infty}+\bar{r}}+\sigma_{w}^{2}
$$

where $\bar{r}$ and $\bar{c}$ are the time-invariant versions of $\bar{r}_{k}$ and $\bar{c}_{k} \cdot{ }^{4}$ For stable systems, it is known that the steady-state error covariance always exists [41, p. 77]. For $\bar{c} \neq 0$, the solution to this can be easily shown to be

$$
P_{\infty}=\frac{\left(a^{2}-1\right) \bar{r}+\bar{c}^{2} \sigma_{w}^{2}+\sqrt{\left(\left(a^{2}-1\right) \bar{r}+\bar{c}^{2} \sigma_{w}^{2}\right)^{2}+4 \bar{c}^{2} \sigma_{w}^{2} \bar{r}}}{2 \bar{c}^{2}} .
$$

In the "degenerate" case where $\bar{c}=0$, we have $P_{\infty}=$ $\sigma_{w}^{2} /\left(1-a^{2}\right)$. It is also usful to write (7) as

$$
P_{\infty}=\frac{a^{2}-1+\sigma_{w}^{2} S+\sqrt{\left(a^{2}-1+\sigma_{w}^{2} S\right)^{2}+4 \sigma_{w}^{2} S}}{2 S}
$$

with $S \equiv \bar{c}^{2} / \bar{r}$ regarded as a signal-to-noise ratio (SNR). We have the following property.

LEMMA $1 \quad P_{\infty}$ as defined by (8) is a decreasing function of $S$.

ProOF See the Appendix.

\footnotetext{
${ }^{4}$ The assumption of time invariance is important. For time-varying $\bar{r}_{k}$ and $\bar{c}_{k}$, the error covariance usually will not converge to a steady-state value.
} 
Similarly, in the orthogonal access scheme, the steady-state error covariance $P_{\infty}^{o}$ satisfies (cf. (5))

$$
P_{\infty}^{o}=\frac{a^{2} P_{\infty}^{o}}{1+P_{\infty}^{o} \overline{\mathbf{C}}^{o^{T}} \overline{\mathbf{R}}^{o^{-1}} \overline{\mathbf{C}}^{o}}+\sigma_{w}^{2}
$$

where $\overline{\mathbf{R}}^{o}$ and $\overline{\mathbf{C}}^{o}$ are the time-invariant versions of $\overline{\mathbf{R}}_{k}^{o}$ and $\overline{\mathbf{C}}_{k}^{o}$. We can easily compute $\overline{\mathbf{C}}^{o^{T}} \overline{\mathbf{R}}^{o^{-1}} \overline{\mathbf{C}}^{o}=$ $\sum_{i=1}^{M} \alpha_{i}^{2} h_{i}^{2} c_{i}^{2} /\left(\alpha_{i}^{2} h_{i}^{2} \sigma_{i}^{2}+\sigma_{n}^{2}\right)$ with $S^{o} \equiv \overline{\mathbf{C}}^{o^{T}} \overline{\mathbf{R}}^{o^{-1}} \overline{\mathbf{C}}^{o}$ regarded as an SNR. The solution to (9) can then be found as

$$
P_{\infty}^{o}=\frac{a^{2}-1+\sigma_{w}^{2} S^{o}+\sqrt{\left(a^{2}-1+\sigma_{w}^{2} S^{o}\right)^{2}+4 \sigma_{w}^{2} S^{o}}}{2 S^{o}} .
$$

LEMMA $2 \quad P_{\infty}^{o}$ as defined by (10) is a decreasing function of $S^{\circ}$.

The proof is the same as that of Lemma 1 in the Appendix.

Comparing (8) and (10), we see that the functions for $P_{\infty}$ and $P_{\infty}^{o}$ are of the same form, except that in the multi-access scheme we have

$$
S \equiv \frac{\bar{c}^{2}}{\bar{r}}=\frac{\left(\sum_{i=1}^{M} \alpha_{i} h_{i} c_{i}\right)^{2}}{\sum_{i=1}^{M} \alpha_{i}^{2} h_{i}^{2} \sigma_{i}^{2}+\sigma_{n}^{2}}
$$

and in the orthogonal scheme we have

$$
S^{o} \equiv \overline{\mathbf{C}}^{o^{T}} \overline{\mathbf{R}}^{o^{-1}} \overline{\mathbf{C}}^{o}=\sum_{i=1}^{M} \frac{\alpha_{i}^{2} h_{i}^{2} c_{i}^{2}}{\alpha_{i}^{2} h_{i}^{2} \sigma_{i}^{2}+\sigma_{n}^{2}} .
$$

E. Some Examples of Multi-Access versus Orthogonal Access

A natural question to ask is whether one scheme always performs better than the other, e.g., whether $S \geq S^{o}$ given the same values for $\alpha_{i}, h_{i}, c_{i}, \sigma_{i}^{2}, \sigma_{n}^{2}$ are used in both expressions. We present below a number of examples to illustrate that in general this is not true. Assume for simplicity that the $\alpha_{i}$ s are chosen such $\alpha_{i} c_{i}$ are positive for all $i=1, \ldots, M$.

1) Consider first the case when $\sigma_{n}^{2}=0$. Then we have the inequality

$$
\sum_{i=1}^{M} \frac{\alpha_{i}^{2} h_{i}^{2} c_{i}^{2}}{\alpha_{i}^{2} h_{i}^{2} \sigma_{i}^{2}} \geq \frac{\left(\sum_{i=1}^{M} \alpha_{i} h_{i} c_{i}\right)^{2}}{\sum_{i=1}^{M} \alpha_{i}^{2} h_{i}^{2} \sigma_{i}^{2}}
$$

which can be shown by applying Theorem 65 of [42]. So when $\sigma_{n}^{2}=0, S^{o} \geq S$ and consequently $P_{\infty}^{o}$ will be smaller than $P_{\infty}$. The intuitive explanation for this is that if there is no noise introduced at the fusion center, then receiving the individual measurements from the sensors is better than receiving a linear combination of the measurements; see also [43].
2) Next we consider the case when the noise variance $\sigma_{n}^{2}$ is large. We can express $S-S^{o}$ as

$$
\begin{aligned}
& \frac{1}{\left(\sum_{i=1}^{M} \alpha_{i}^{2} h_{i}^{2} \sigma_{i}^{2}+\sigma_{n}^{2}\right) \prod_{i=1}^{M}\left(\alpha_{i}^{2} h_{i}^{2} \sigma_{i}^{2}+\sigma_{n}^{2}\right)} \\
& \times\left(\left(\sum_{i=1}^{M} \alpha_{i} h_{i} c_{i}\right)^{2} \prod_{i=1}^{M}\left(\alpha_{i}^{2} h_{i}^{2} \sigma_{i}^{2}+\sigma_{n}^{2}\right)\right. \\
& \quad-\alpha_{1}^{2} h_{1}^{2} c_{1}^{2}\left(\sum_{i=1}^{M} \alpha_{i}^{2} h_{i}^{2} \sigma_{i}^{2}+\sigma_{n}^{2}\right) \prod_{i: i \neq 1}\left(\alpha_{i}^{2} h_{i}^{2} \sigma_{i}^{2}+\sigma_{n}^{2}\right)-\cdots \\
& \left.\quad-\alpha_{M}^{2} h_{M}^{2} c_{M}^{2}\left(\sum_{i=1}^{M} \alpha_{i}^{2} h_{i}^{2} \sigma_{i}^{2}+\sigma_{n}^{2}\right) \prod_{i: i \neq M}\left(\alpha_{i}^{2} h_{i}^{2} \sigma_{i}^{2}+\sigma_{n}^{2}\right)\right) .
\end{aligned}
$$

The coefficient of the $\left(\sigma_{n}^{2}\right)^{M}$ term in the numerator is $\left(\sum_{i=1}^{M} \alpha_{i} h_{i} c_{i}\right)^{2}-\alpha_{1}^{2} h_{1}^{2} c_{1}^{2}-\cdots-\alpha_{M}^{2} h_{M}^{2} c_{M}^{2}>0$. When $\sigma_{n}^{2}$ is sufficiently large, this term will dominate, hence $S>S^{o}$ and the multi-access scheme will now have smaller error covariance than the orthogonal scheme.

3) Now we consider the "symmetric" situation where $\alpha_{i}=\alpha, c_{i}=c, \sigma_{i}^{2}=\sigma_{v}^{2}, h_{i}=h, \forall i$. Then we have

$$
S=\frac{M^{2} \alpha^{2} h^{2} c^{2}}{M \alpha^{2} h^{2} \sigma_{v}^{2}+\sigma_{n}^{2}}=\frac{M \alpha^{2} h^{2} c^{2}}{\alpha^{2} h^{2} \sigma_{v}^{2}+\sigma_{n}^{2} / M}
$$

and

$$
S^{o}=\frac{M \alpha^{2} h^{2} c^{2}}{\alpha^{2} h^{2} \sigma_{v}^{2}+\sigma_{n}^{2}} .
$$

Hence $S \geq S^{o}$, with equality only when $\sigma_{n}^{2}=0$ (or $M=1$ ). Thus in the symmetric case, the multi-access scheme outperforms the orthogonal access scheme.

4) Suppose $\sigma_{n}^{2} \neq 0$. We wish to know whether it is always the case that $S>S^{o}$ for $M$ sufficiently large. The following counterexample shows that in general this assertion is false. Let $\alpha_{i}=1, h_{i}=1, \sigma_{i}^{2}=1, \forall i$. Let $M / 2$ of the sensors have $c_{i}=1$ and the other $M / 2$ sensors have $c_{i}=2$. We find that

$$
S=\frac{(M / 2+M)^{2}}{M+\sigma_{n}^{2}}=\frac{9}{4} \frac{M}{1+\sigma_{n}^{2} / M}
$$

and

$$
S^{o}=\frac{M}{2} \frac{1+4}{1+\sigma_{n}^{2}}=\frac{5}{2} \frac{M}{1+\sigma_{n}^{2}} .
$$

If, e.g., $\sigma_{n}^{2}=1 / 8$, then it may be verified that $S^{o}>S$ for $M<10, S^{o}=S$ for $M=10$, and $S>S^{o}$ for $M>10$, so eventually the multi-access scheme outperforms the orthogonal scheme. On the other hand, if $5 /\left(2\left(1+\sigma_{n}^{2}\right)\right)>9 / 4$ or $\sigma_{n}^{2}<1 / 9$, we will have $S^{o}>S$ no matter how large $M$ is.

\section{ASYMPTOTIC BEHAVIOUR}

Since $P_{\infty}$ is a decreasing function of $S$ (similar comments apply for the orthogonal scheme), increasing $S$ will provide an improvement in performance. As $S \rightarrow \infty$, we can see from (8) that 
$P_{\infty} \rightarrow \sigma_{w}^{2}$, the process noise variance. Note that unlike, e.g., [15], [24], where the mean squared error (MSE) can be driven to zero in situations such as when there are a large number of sensors, here the lower bound $\sigma_{w}^{2}$ on performance is always strictly greater than zero. When the number of sensors is fixed, then it is not too difficult to show that $S$ will be bounded no matter how large (or small) one makes the $\alpha_{i}$ s, so getting arbitrarily close to $\sigma_{w}^{2}$ is not possible. On the other hand, if instead the number of sensors $M$ is allowed to increase, then $P_{\infty} \rightarrow \sigma_{w}^{2}$ as $M \rightarrow \infty$ can be achieved in many situations, as shown in the following. Moreover we are interested in the rate at which this convergence occurs.

In this section we will first investigate two simple strategies, 1) $\alpha_{i}=1, \forall i$, and 2) $\alpha_{i}=1 / \sqrt{M}, \forall i{ }^{5}$ For the "symmetric" case (i.e., the parameters are the same for each sensor), we obtain explicit asymptotic expressions. We then use these results to bound the performance in the general asymmetric case in Section IIIC. Finally, we also investigate the asymptotic performance of a simple equal-power allocation scheme in Section IIID. We note that the results in this section assume that large $M$ is possible, e.g., ability to synchronize a large number of sensors in the multi-access scheme, or the availability of a large number of orthogonal channels in the orthogonal scheme, which may not always be the case in practice. On the other hand, in numerical investigations we have found that the results derived in this section are quite accurate even for 20-30 sensors, see Figs. 1 and 2 in Section V.

\section{A. No Scaling: $\alpha_{i}=1, \forall i$}

Let $\alpha_{i}=1, \forall i$, so measurements are forwarded to the fusion center without any scaling. Assume for simplicity the symmetric case, where $c_{i}=c, \sigma_{i}^{2}=\sigma_{v}^{2}$, $h_{i}=h, \forall i$.

In the multi-access scheme, $\bar{c}=M h c$, and $\bar{v}_{k}$ has variance $\bar{r}=M h^{2} \sigma_{v}^{2}+\sigma_{n}^{2}$, so that $S=$ $M^{2} h^{2} c^{2} /\left(M h^{2} \sigma_{v}^{2}+\sigma_{n}^{2}\right)$. Since $S \rightarrow \infty$ as $M \rightarrow \infty$, we have by the previous discussion that $P_{\infty} \rightarrow \sigma_{w}^{2}$. The rate of convergence is given by the following:

LEMMA 3 In the symmetric multi-access scheme with $\alpha_{i}=1, \forall i$,

$$
P_{\infty}=\sigma_{w}^{2}+\frac{a^{2} \sigma_{v}^{2}}{c^{2}} \frac{1}{M}+O\left(\frac{1}{M^{2}}\right)
$$

as $M \rightarrow \infty$.

Proof See the Appendix.

\footnotetext{
${ }^{5}$ These strategies are similar to the case of "equal power constraint" and "total power constraint" in [44] (also [16]), and various versions have also been considered in the work of [15], [23]-[25], in the context of estimation of IID processes.
}

Thus the steady-state error covariance for the multi-access scheme converges to the process noise variance $\sigma_{w}^{2}$ at a rate of $1 / M$. This result matches the rate of $1 / M$ achieved for estimation of IID processes using multi-access schemes, e.g., [15, 44].

In the orthogonal scheme we have $S^{o}=$ $M h^{2} c^{2} /\left(h^{2} \sigma_{v}^{2}+\sigma_{n}^{2}\right)$, so $S^{o} \rightarrow \infty$ as $M \rightarrow \infty$ also. By calculations similar to the proof of Lemma 3 we find that as $M \rightarrow \infty$,

$$
\begin{aligned}
P_{\infty}^{o} & =\sigma_{w}^{2}+\frac{a^{2}\left(h^{2} \sigma_{v}^{2}+\sigma_{n}^{2}\right)}{h^{2} c^{2}} \frac{1}{M}+O\left(\frac{1}{M^{2}}\right) \\
& =\sigma_{w}^{2}+\frac{a^{2}\left(\sigma_{v}^{2}+\sigma_{n}^{2} / h^{2}\right)}{c^{2}} \frac{1}{M}+O\left(\frac{1}{M^{2}}\right) .
\end{aligned}
$$

Therefore, the steady-state error covariance again converges to $\sigma_{w}^{2}$ at a rate of $1 / M$, but the constant $a^{2}\left(\sigma_{v}^{2}+\sigma_{n}^{2} / h^{2}\right) / c^{2}$ in front is larger. This agrees with example 3 of Section IIE that, in the symmetric situation, the multi-access scheme will perform better than the orthogonal scheme.

\section{B. Scaling $\alpha_{i}=1 / \sqrt{M}, \forall i$}

In the previous case with $\alpha_{i}=1, \forall i$, the power received at the fusion center will grow unbounded as $M \rightarrow \infty$. Suppose instead we let $\alpha_{i}=1 / \sqrt{M}$, $\forall i$, which will keep the power received at the fusion center bounded (and is constant in the symmetric case), while the transmit power used by each sensor will tend to zero as $M \rightarrow \infty$. Again assume for simplicity that $c_{i}=c, \sigma_{i}^{2}=\sigma_{v}^{2}, h_{i}=h$, $\forall i$.

In the multi-access scheme, we now have $S=$ $M h^{2} c^{2} /\left(h^{2} \sigma_{v}^{2}+\sigma_{n}^{2}\right)$, so that as $M \rightarrow \infty$,

$$
P_{\infty}=\sigma_{w}^{2}+\frac{a^{2}\left(\sigma_{v}^{2}+\sigma_{n}^{2} / h^{2}\right)}{c^{2}} \frac{1}{M}+O\left(\frac{1}{M^{2}}\right) .
$$

Thus we again have the steady-state error covariance converging to the process noise variance $\sigma_{w}^{2}$ at a rate of $1 / M$. In fact, we see that this is the same expression as (12) in the orthogonal scheme, but there we were using $\alpha_{i}=1, \forall i$. The difference here is that this performance can be achieved even when the transmit power used by each individual sensor will decrease to zero as the number of sensors increases, which can be quite desirable in power-constrained environments such as wireless sensor networks. For IID processes, this somewhat surprising behaviour when the total received power is bounded has also been observed $[25,44]$.

In the orthogonal scheme we have $S^{o}=$ $h^{2} c^{2} /\left(h^{2} \sigma_{v}^{2} / M+\sigma_{n}^{2}\right)$, and we note that now $S^{o}$ is bounded even as $M \rightarrow \infty$, so $P_{\infty}^{o}$ cannot converge to $\sigma_{w}^{2}$ as $M \rightarrow \infty$. For a more precise expression, we can show by similar computations to the proof of 
Lemma 3 that for large $M$,

$$
\begin{aligned}
P_{\infty}^{o}= & \frac{\left(a^{2}-1\right) \sigma_{n}^{2}+h^{2} c^{2} \sigma_{w}^{2}+\sqrt{\left(a^{2}-1\right)^{2} \sigma_{n}^{4}+2\left(a^{2}+1\right) \sigma_{n}^{2} h^{2} c^{2} \sigma_{w}^{2}+h^{4} c^{4} \sigma_{w}^{4}}}{2 h^{2} c^{2}} \\
& +\left[\frac{\left(a^{2}-1\right) \sigma_{v}^{2}}{2 c^{2}}+\frac{\left(a^{2}+1\right) h^{4} \sigma_{v}^{2} c^{2} \sigma_{w}^{2}+\left(a^{2}-1\right)^{2} \sigma_{n}^{2} h^{2} \sigma_{v}^{2}}{2 h^{2} c^{2} \sqrt{\left(a^{2}-1\right)^{2} \sigma_{n}^{4}+2\left(a^{2}+1\right) \sigma_{n}^{2} h^{2} c^{2} \sigma_{w}^{2}+h^{4} c^{4} \sigma_{w}^{4}}}\right] \frac{1}{M}+O\left(\frac{1}{M^{2}}\right) .
\end{aligned}
$$

Noting that

$$
\frac{\left(a^{2}-1\right) \sigma_{n}^{2}+h^{2} c^{2} \sigma_{w}^{2}+\sqrt{\left(a^{2}-1\right)^{2} \sigma_{n}^{4}+2\left(a^{2}+1\right) \sigma_{n}^{2} h^{2} c^{2} \sigma_{w}^{2}+h^{4} c^{4} \sigma_{w}^{4}}}{2 h^{2} c^{2}}>\sigma_{w}^{2}
$$

the steady-state error covariance will converge as $M \rightarrow \infty$ to a value strictly greater than $\sigma_{w}^{2}$, though the convergence is still at a rate $1 / M$. Analogously for IID processes, it has been shown that in the orthogonal scheme the MSE does not go to zero as $M \rightarrow \infty$ when the total power used is bounded [24].

\section{General Parameters}

The behaviour shown in the two previous cases can still hold under more general conditions on $c_{i}, \sigma_{i}^{2}$, and $h_{i}$. Suppose for instance that they can be bounded from both above and below, i.e., $0<c_{\min } \leq\left|c_{i}\right| \leq$ $c_{\max }<\infty, 0<\sigma_{\min }^{2} \leq \sigma_{i}^{2} \leq \sigma_{\max }^{2}<\infty, 0<h_{\min } \leq h_{i} \leq$ $h_{\max }<\infty, \forall i$. We have the following:

LEMMA 4 In the general multi-access scheme, as $M \rightarrow \infty$, using either no scaling of measurements, or scaling of measurements by $1 / \sqrt{M}$, results in

$$
P_{\infty}=\sigma_{w}^{2}+O\left(\frac{1}{M}\right) .
$$

In the general orthogonal scheme, using no scaling of measurements results in

$$
P_{\infty}^{o}=\sigma_{w}^{2}+O\left(\frac{1}{M}\right)
$$

as $M \rightarrow \infty$, but $P_{\infty}^{o}$ does not converge to a limit (in general) as $M \rightarrow \infty$ when measurements are scaled by $1 / \sqrt{M}$.

ProOF See the Appendix.

\section{Asymptotic Behaviour under Equal-Power} Allocation

When the parameters are asymmetric, the above rules will in general allocate different powers to the individual sensors. Another simple alternative is to use equal-power allocation. Recall that the transmit power used by each sensor is $\gamma_{i}=\alpha_{i}^{2}\left(c_{i}^{2} \sigma_{w}^{2} /\left(1-a^{2}\right)+\sigma_{i}^{2}\right)$. If we allocate power $\gamma$ to each sensor, i.e., $\gamma_{i}=\gamma, \forall i$, then

$$
\alpha_{i}=\sqrt{\frac{\gamma\left(1-a^{2}\right)}{c_{i}^{2} \sigma_{w}^{2}+\sigma_{i}^{2}\left(1-a^{2}\right)}} .
$$

If instead the total power $\gamma_{\text {total }}$ is to be shared equally amongst sensors, then $\gamma_{i}=\gamma_{\text {total }} / M, \forall i$, and

$$
\alpha_{i}=\sqrt{\frac{\gamma_{\text {total }}\left(1-a^{2}\right)}{M\left(c_{i}^{2} \sigma_{w}^{2}+\sigma_{i}^{2}\left(1-a^{2}\right)\right)}} .
$$

Asymptotic results under equal-power allocation are quite similar to Section IIIC, namely,

LEMMA 5 In the general multi-access scheme, as $M \rightarrow \infty$, using the equal-power allocation (15) or (16) results in

$$
P_{\infty}=\sigma_{w}^{2}+O\left(\frac{1}{M}\right) .
$$

In the general orthogonal scheme, using the equal-power allocation (15) results in

$$
P_{\infty}^{o}=\sigma_{w}^{2}+O\left(\frac{1}{M}\right)
$$

as $M \rightarrow \infty$, but $P_{\infty}^{o}$ does not converge to a limit as $M \rightarrow \infty$ when using the power allocation (16).

Proof See the Appendix.

\section{E. Remarks}

1) Most of the previous policies in this section give a convergence rate of $1 / \mathrm{M}$. We might wonder whether one can achieve an even better rate (e.g., $1 / M^{2}$ ) using other choices for $\alpha_{i}$, though the answer turns out to be no. To see this, following [15], consider the "ideal" case where sensor measurements are received perfectly at the fusion center, and which mathematically corresponds to the orthogonal scheme with $\sigma_{n}^{2}=0, \alpha_{i}=1, h_{i}=1, \forall i$. This idealized situation provides a lower bound on the achievable error covariance. We will have $S^{o}=\sum_{i=1}^{M} c_{i}^{2} / \sigma_{i}^{2}$, which can then be used to show that $P_{\infty}^{o}$ converges to $\sigma_{w}^{2}$ at the rate $1 / M$. Hence $1 / M$ is the best rate that can be achieved with any coded/uncoded scheme.

2) In the previous derivations we have not actually used the assumption that $|a|<1$, so the results in Sections IIIA-IIIC will hold even when the system is unstable (assuming $\bar{C} \neq 0$ ). However for unstable systems, $\mathbb{E}\left[x_{k}^{2}\right]$ becomes unbounded as $k \rightarrow \infty$, so 
if the $\alpha_{i, k} \mathrm{~s}$ are time invariant, then more and more power is used by the sensors as time passes. If the application is a wireless sensor network where power is limited, then the question is whether one can choose these $\alpha_{i, k}$ s such that both the power used by the sensors and the error covariances will be bounded for all times. Now if there is no noise at the fusion center, i.e., $n_{k}=0$, then a simple scaling of the measurements at the individual sensors will work. But when $n_{k} \neq 0$, as will usually be the case in analog forwarding, we have not been able to find a scheme that can achieve this. Note however that for unstable systems, asymptotic results are of mathematical interest only. In practice (in most cases) we will be interested in finite horizon results for unstable systems where the system states and measurements can take on large values but are still bounded. In such finite horizon situations, one can perform optimum power allocation at each time step similar to the process presented in Section IVD but for a finite number of time steps. One can also use a finite horizon dynamic programming approach similar to Section IVD4. However these problems not addressed in the current paper.

\section{OPTIMAL POWER ALLOCATION}

When there are a large number of sensors, one can use simple strategies such as $\alpha_{i}=1 / \sqrt{M}$, $\forall i$ or the equal power allocation (16), which will both give a convergence of the steady-state error covariance to $\sigma_{w}^{2}$ at a rate of $1 / M$ in the multi-access scheme, while bounding the total power used by all the sensors. However when the number of sensors is small, one may perhaps do better with different choices of the $\alpha_{i} \mathrm{~s}$. In this section we study some relevant power allocation problems. These are considered first for static channels in the multi-access and orthogonal schemes in Sections IVA and IVB, respectively. Some features of the solutions to these optimization problems are discussed in Section IVC. These results are then extended to fading channels with CSI and fading channels without CSI in Sections IVD and IVE, respectively.

\section{A. Optimization Problems for Multi-Access Scheme}

1) Minimizing Sum Power: One possible formulation is to minimize the sum of transmit powers used by the sensors subject to a bound $D$ on the steady-state error covariance. More formally, the problem is

$$
\begin{aligned}
& \min \sum_{i=1}^{M} \gamma_{i}=\sum_{i=1}^{M} \alpha_{i}^{2}\left(\frac{c_{i}^{2} \sigma_{w}^{2}}{1-a^{2}}+\sigma_{i}^{2}\right) \\
& \text { subject to } P_{\infty} \leq D
\end{aligned}
$$

with $P_{\infty}$ given by (7). Some straightforward manipulations show that the constraint can be simplified to

i.e.,

$$
\bar{r}\left(a^{2} D+\sigma_{w}^{2}-D\right)+\bar{c}^{2} D\left(\sigma_{w}^{2}-D\right) \leq 0
$$

$$
\begin{gathered}
\left(\sum_{i=1}^{M} \alpha_{i}^{2} h_{i}^{2} \sigma_{i}^{2}+\sigma_{n}^{2}\right)\left(a^{2} D+\sigma_{w}^{2}-D\right) \\
+\left(\sum_{i=1}^{M} \alpha_{i} h_{i} c_{i}\right)^{2} D\left(\sigma_{w}^{2}-D\right) \leq 0 .
\end{gathered}
$$

Now define $s=h_{1} c_{1} \alpha_{1}+\cdots+h_{M} c_{M} \alpha_{M}$. Then the optimization problem becomes

$$
\begin{aligned}
& \min _{\alpha_{1}, \ldots, \alpha_{M}, s} \sum_{i=1}^{M} \alpha_{i}^{2}\left(\frac{c_{i}^{2} \sigma_{w}^{2}}{1-a^{2}}+\sigma_{i}^{2}\right) \\
& \text { subject to }\left(\sum_{i=1}^{M} \alpha_{i}^{2} h_{i}^{2} \sigma_{i}^{2}+\sigma_{n}^{2}\right)\left(a^{2} D+\sigma_{w}^{2}-D\right) \\
& \leq s^{2} D\left(D-\sigma_{w}^{2}\right) \quad \text { and } \quad s=\sum_{i=1}^{M} h_{i} c_{i} \alpha_{i} .
\end{aligned}
$$

Before continuing further, let us first determine some upper and lower bounds on $D$. From Section III, a lower bound is $D \geq \sigma_{w}^{2}$, the process noise variance. For an upper bound, suppose $\bar{c}=0$ so we don't have any information about $x_{k}$. Since we are assuming the system is stable, one can still achieve an error covariance of $\sigma_{w}^{2} /\left(1-a^{2}\right)$ (e.g., just let $\hat{x}_{k}=0, \forall k$ ), so $D \leq \sigma_{w}^{2} /\left(1-a^{2}\right)$. Hence in problem (18) both $D-\sigma_{w}^{2}$ and $a^{2} D+\sigma_{w}^{2}-D$ are positive quantities.

To reduce the amount of repetition in later sections, consider the slightly more general problem

$$
\min _{\alpha_{1}, \ldots, \alpha_{M}, s} \sum_{i=1}^{M} \alpha_{i}^{2} \kappa_{i}
$$

subject to $\left(\sum_{i=1}^{M} \alpha_{i}^{2} \tau_{i}+\sigma_{n}^{2}\right) x \leq s^{2} y \quad$ and $\quad s=\sum_{i=1}^{M} \alpha_{i} \rho_{i}$

where $x>0, y>0, \kappa_{i}>0, \rho_{i} \in \mathbb{R}, \tau_{i}>0, i=1, \ldots, M$ are constants. In the context of (18), $x=a^{2} D+$ $\sigma_{w}^{2}-D, y=D\left(D-\sigma_{w}^{2}\right), \rho_{i}=h_{i} c_{i}, \tau_{i}=h_{i}^{2} \sigma_{i}^{2}$ and $\kappa_{i}=$ $c_{i}^{2} \sigma_{w}^{2} /\left(1-a^{2}\right)+\sigma_{i}^{2}$ for $i=1, \ldots, M$.

The objective function of problem (19) is clearly convex. Noting that $\tau_{i}, \sigma_{n}^{2}, x$ and $y$ are all positive, the set of points satisfying $\left(\sum_{i=1}^{M} \tau_{i} \alpha_{i}^{2}+\sigma_{n}^{2}\right) x=y s^{2}$ is then a quadric surface that consists of two pieces, corresponding to $s>0$ and $s<0 .{ }^{6}$ Furthermore, the set of points satisfying $\left(\sum_{i=1}^{M} \tau_{i} \alpha_{i}^{2}+\sigma_{n}^{2}\right) x \leq y s^{2}$ and $s>$ 0 , and the set of points satisfying $\left(\sum_{i=1}^{M} \tau_{i} \alpha_{i}^{2}+\sigma_{n}^{2}\right) x \leq$

\footnotetext{
${ }^{6}$ In three dimensions this surface corresponds to a "hyperboloid of two sheets."
} 
$y s^{2}$ and $s<0$, are both known to be convex sets, see e.g., Proposition 15.4.7 of [45]. Hence the parts of the feasible region corresponding to $s>0$ and $s<0$ are both convex, and the global solution can be efficiently obtained numerically. Furthermore, following similar steps to [23], a solution in (mostly) closed form can actually be obtained. We omit the derivations but summarise what is required.

One first solves numerically for $\lambda$ the equation

$$
\sum_{i=1}^{M} \frac{\lambda \rho_{i}^{2}}{\kappa_{i}+\lambda \tau_{i} x}=\frac{1}{y} .
$$

Since the left-hand side is increasing with $\lambda$, solutions to this equation will be unique provided it exists.

Taking limits as $\lambda \rightarrow \infty$, we see that a solution exists if and only if

$$
\sum_{i=1}^{M} \frac{\rho_{i}^{2}}{\tau_{i}}>\frac{x}{y}
$$

Equation (20) thus provides a feasibility check for the optimization problem (19). In the context of (18), one can easily derive that (20) implies

$$
\sum_{i=1}^{M} \frac{c_{i}^{2}}{\sigma_{i}^{2}}>\frac{a^{2} D+\sigma_{w}^{2}-D}{D\left(D-\sigma_{w}^{2}\right)}
$$

which indicates that the sum of the sensor SNRs must be greater than a threshold (dependent on the error covariance threshold $D$ ) for the optimization problem (18) to be feasible. Next, we compute $\mu$ from

$$
\mu^{2}=\sigma_{n}^{2} x\left(\sum_{i=1}^{M} \frac{\rho_{i}^{2} \kappa_{i}}{4 \lambda\left(\kappa_{i}+\lambda \tau_{i} x\right)^{2}}\right)^{-1} .
$$

Finally we obtain the optimal $\alpha_{i}$ s (denoted by $\alpha_{i}^{*}$ )

$$
\alpha_{i}^{*}=\frac{\mu \rho_{i}}{2\left(\kappa_{i}+\lambda \tau_{i} x\right)}, \quad i=1, \ldots, M
$$

with the resulting powers

$$
\gamma_{i}=\alpha_{i}^{* 2} \kappa_{i}=\alpha_{i}^{* 2}\left(c_{i}^{2} \frac{\sigma_{w}^{2}}{1-a^{2}}+\sigma_{i}^{2}\right), \quad i=1, \ldots, M .
$$

Note that depending on whether we choose $\mu$ to be positive or negative, two different sets of $\alpha_{i}^{*}$ s are obtained, one of which is the negative of the other, though the $\gamma_{i}$ s and hence the optimal value of the objective function remains the same.

Another interesting relation that can be shown (see [23]) is that the optimal sum power satisfies

$$
\gamma_{\text {total }}^{*}=\sum_{i=1}^{M} \alpha_{i}^{* 2} \kappa_{i}=\lambda \sigma_{n}^{2} x .
$$

This relation is useful in obtaining an analytic solution to problem (23) next.

2) Minimizing Error Covariance: A related problem is to minimize the steady-state error covariance subject to a sum power constraint $\gamma_{\text {total }}$. Formally, this is

$$
\begin{aligned}
& \min P_{\infty} \\
& \text { subject to } \sum_{i=1}^{M} \alpha_{i}^{2}\left(\frac{c_{i}^{2} \sigma_{w}^{2}}{1-a^{2}}+\sigma_{i}^{2}\right) \leq \gamma_{\text {total }}
\end{aligned}
$$

with $P_{\infty}$ again given by (7). For this problem, the feasible region is clearly convex, but the objective function is complicated. To simplify the objective, recall from Lemma 1 that $P_{\infty}$ is a decreasing function of $S=\bar{c}^{2} / \bar{r}$. Thus maximizing $\bar{c}^{2} / \bar{r}$ (or minimizing $\left.\bar{r} / \bar{c}^{2}\right)$ is equivalent to minimizing $P_{\infty}$, which can be interpreted as maximizing the SNR minimizes $P_{\infty}$. Hence the problem is equivalent to

$$
\begin{gathered}
\min _{\alpha_{1}, \ldots, \alpha_{M}, s} \frac{\sum_{i=1}^{M} \alpha_{i}^{2} h_{i}^{2} \sigma_{i}^{2}+\sigma_{n}^{2}}{s^{2}} \\
\text { subject to } \quad \sum_{i=1}^{M} \alpha_{i}^{2}\left(\frac{c_{i}^{2} \sigma_{w}^{2}}{1-a^{2}}+\sigma_{i}^{2}\right) \leq \gamma_{\text {total }} \\
\text { and } s=\sum_{i=1}^{M} h_{i} c_{i} \alpha_{i} .
\end{gathered}
$$

We again introduce a more general problem:

$$
\begin{aligned}
\min _{\alpha_{1}, \ldots, \alpha_{M}, s} \frac{\sum_{i=1}^{M} \alpha_{i}^{2} \tau_{i}+\sigma_{n}^{2}}{s^{2}} \\
\text { subject to } \sum_{i=1}^{M} \alpha_{i}^{2} \kappa_{i} \leq \gamma_{\text {total }} \text { and } s=\sum_{i=1}^{M} \alpha_{i} \rho_{i}
\end{aligned}
$$

with $x>0, y>0, \kappa_{i}>0, \rho_{i} \in \mathbb{R}, \tau_{i}>0, i=1, \ldots, M$ being constants. The objective function is still nonconvex; however by making use of the properties of the analytical solution to problem (19), such as the relation (22), an analytical solution to problem (23) can also be obtained. The optimal $\alpha_{i} \mathrm{~s}$ can be shown to satisfy

$$
\begin{aligned}
\alpha_{i}^{* 2}= & \gamma_{\text {total }}\left(\sum_{j=1}^{M} \frac{\rho_{j}^{2}}{\left(\kappa_{j}+\gamma_{\text {total }} \frac{\tau_{j}}{\sigma_{n}^{2}}\right)^{2}} \kappa_{j}\right)^{-1} \\
& \times \frac{\rho_{i}^{2}}{\left(\kappa_{i}+\gamma_{\text {total }} \frac{\tau_{i}}{\sigma_{n}^{2}}\right)^{2}} \kappa_{i} .
\end{aligned}
$$

The details on obtaining this solution are similar to those in [23] and are omitted.

B. Optimization Problems for Orthogonal Access Scheme

1) Minimizing Sum Power: The corresponding problem of minimizing the sum power in the 
orthogonal scheme is

$$
\begin{aligned}
& \min \sum_{i=1}^{M} \gamma_{i}=\sum_{i=1}^{M} \alpha_{i}^{2}\left(\frac{c_{i}^{2} \sigma_{w}^{2}}{1-a^{2}}+\sigma_{i}^{2}\right) \\
& \text { subject to } P_{\infty}^{o} \leq D
\end{aligned}
$$

with $P_{\infty}^{o}$ now given by (10). By a rearrangement of the constraint, this can be shown to be equivalent to

$$
\begin{aligned}
& \min _{\alpha_{1}^{2}, \ldots, \alpha_{M}^{2}} \sum_{i=1}^{M} \alpha_{i}^{2}\left(\frac{c_{i}^{2} \sigma_{w}^{2}}{1-a^{2}}+\sigma_{i}^{2}\right) \\
& \text { subject to } \sum_{i=1}^{M} \frac{\alpha_{i}^{2} h_{i}^{2} c_{i}^{2}}{\alpha_{i}^{2} h_{i}^{2} \sigma_{i}^{2}+\sigma_{n}^{2}} \geq \frac{a^{2} D+\sigma_{w}^{2}-D}{D\left(D-\sigma_{w}^{2}\right)} .
\end{aligned}
$$

Note that in contrast to the multi-access scheme, we now write the minimization over $\alpha_{i}^{2}$ rather than $\alpha_{i}$. Since each of the functions

$$
\frac{-\alpha_{i}^{2} h_{i}^{2} c_{i}^{2}}{\alpha_{i}^{2} h_{i}^{2} \sigma_{i}^{2}+\sigma_{n}^{2}}=\frac{-c_{i}^{2}}{\sigma_{i}^{2}}+\frac{\sigma_{n}^{2} c_{i}^{2} / \sigma_{i}^{2}}{\alpha_{i}^{2} h_{i}^{2} \sigma_{i}^{2}+\sigma_{n}^{2}}
$$

is convex in $\alpha_{i}^{2}$, the problem is a convex optimization problem in $\left(\alpha_{1}^{2}, \ldots, \alpha_{M}^{2}\right)$. Note that without further restrictions on $\alpha_{i}$, we get $2^{M}$ solutions with the same values of the objective function corresponding to the different choices of positive and negative signs on the $\alpha_{i} \mathrm{~s}$. This is in contrast to the multi-access scheme where there were two sets of solutions. For simplicity we can take the solution corresponding to all $\alpha_{i} \geq 0$. ${ }^{7}$

An analytical solution can also be obtained. To reduce repetition in later sections, consider the more general problem

$$
\begin{aligned}
& \min _{\alpha_{1}^{2}, \ldots, \alpha_{M}^{2}} \sum_{i=1}^{M} \alpha_{i}^{2} \kappa_{i} \\
& \text { subject to } \sum_{i=1}^{M} \frac{\alpha_{i}^{2} \rho_{i}^{2}}{\alpha_{i}^{2} \tau_{i}+\sigma_{n}^{2}} \geq \frac{x}{y}
\end{aligned}
$$

where $x>0, y>0, \kappa_{i}>0, \rho_{i} \in \mathbb{R}, \tau_{i}>0, i=1, \ldots, M$ are constants and have similar interpretations as in Section IVA1. Since the derivation of the analytical solution is similar to that found in [24] (though what they regard as $\alpha_{k}$ is $\alpha_{i}^{2}$ here), it is omitted, and we only present the solution.

Firstly the problem will be feasible if and only if

$$
\sum_{i=1}^{M} \frac{\rho_{i}^{2}}{\tau_{i}}>\frac{x}{y}
$$

Interestingly this is the same as the feasibility condition (20) for problem (19) in the multi-access scheme, indicating that the total SNR for the sensor

\footnotetext{
${ }^{7}$ In general this is not possible in the multi-access scheme. For instance, if we have two sensors with $c_{1}$ being positive and $c_{2}$ negative, the optimal solution will involve $\alpha_{1}$ being positive and $\alpha_{2}$ negative or vice versa. Restricting both $\alpha_{i}$ s to be positive in the multi-access scheme will result in a suboptimal solution.
}

measurements must be greater than a certain threshold (dependent on $D$ ). The optimal $\alpha_{i}$ s satisfy

$$
\alpha_{i}^{* 2}=\frac{1}{\tau_{i}}\left(\sqrt{\frac{\lambda \rho_{i}^{2} \sigma_{n}^{2}}{\kappa_{i}}}-\sigma_{n}^{2}\right)^{+}
$$

where $(x)^{+}$is the function that is equal to $x$ when $x$ is positive, and zero otherwise. To determine $\lambda$, now assume that the sensors are ordered such that

$$
\frac{\rho_{1}^{2}}{\kappa_{1}} \geq \cdots \geq \frac{\rho_{M}^{2}}{\kappa_{M}} .
$$

Note that in the context of problem (25),

$$
\frac{\rho_{i}^{2}}{\kappa_{i}}=\frac{h_{i}^{2}}{\sigma_{w}^{2} /\left(1-a^{2}\right)+\sigma_{i}^{2} / c_{i}^{2}} .
$$

Clearly, this ordering favours the sensors with better channels and higher measurement quality. Then the optimal values of $\alpha_{i}^{2}$ (and hence $\alpha_{i}^{*}$ ) can also be expressed as

$$
\alpha_{i}^{* 2}= \begin{cases}\frac{1}{\tau_{i}}\left(\sqrt{\frac{\lambda \rho_{i}^{2} \sigma_{n}^{2}}{\kappa_{i}}}-\sigma_{n}^{2}\right), & i \leq M_{1} \\ 0, & \text { otherwise }\end{cases}
$$

where

$$
\sqrt{\lambda}=\frac{\sum_{i=1}^{M_{1}} \frac{\left|\rho_{i}\right|}{\tau_{i}} \sqrt{\kappa_{i} \sigma_{n}^{2}}}{\sum_{i=1}^{M_{1}} \frac{\rho_{i}^{2}}{\tau_{i}}-\frac{x}{y}}
$$

and the number of sensors which are active, $M_{1}$ (which can be shown to be unique [6]), satisfies

$$
\sum_{i=1}^{M_{1}} \frac{\rho_{i}^{2}}{\tau_{i}}-\frac{x}{y} \geq 0, \quad \frac{\sum_{i=1}^{M_{1}} \frac{\left|\rho_{i}\right|}{\tau_{i}} \sqrt{\kappa_{i} \sigma_{n}^{2}}}{\sum_{i=1}^{M_{1}} \frac{\rho_{i}^{2}}{\tau_{i}}-\frac{x}{y}} \sqrt{\frac{\rho_{M_{1}}^{2} \sigma_{n}^{2}}{\kappa_{M_{1}}}}-\sigma_{n}^{2}>0
$$

and

$$
\frac{\sum_{i=1}^{M_{1}+1} \frac{\left|\rho_{i}\right|}{\tau_{i}} \sqrt{\kappa_{i} \sigma_{n}^{2}}}{\sum_{i=1}^{M_{1}+1} \frac{\rho_{i}^{2}}{\tau_{i}}-\frac{x}{y}} \sqrt{\frac{\rho_{M_{1}+1}^{2} \sigma_{n}^{2}}{\kappa_{M_{1}+1}}}-\sigma_{n}^{2} \leq 0 .
$$

2) Minimizing Error Covariance: The corresponding problem of minimizing the error covariance in the orthogonal scheme is equivalent to

$$
\begin{aligned}
& \min _{\alpha_{1}^{2}, \ldots, \alpha_{M}^{2}}-\sum_{i=1}^{M} \frac{\alpha_{i}^{2} h_{i}^{2} c_{i}^{2}}{\alpha_{i}^{2} h_{i}^{2} \sigma_{i}^{2}+\sigma_{n}^{2}} \\
& \text { subject to } \sum_{i=1}^{M} \alpha_{i}^{2}\left(\frac{c_{i}^{2} \sigma_{w}^{2}}{1-a^{2}}+\sigma_{i}^{2}\right) \leq \gamma_{\text {total }}
\end{aligned}
$$

which is again a convex problem in $\left(\alpha_{1}^{2}, \ldots, \alpha_{M}^{2}\right)$. For an analytical solution [24], consider a more general 
problem:

$$
\begin{gathered}
\min _{\alpha_{1}^{2}, \ldots, \alpha_{M}^{2}}-\sum_{i=1}^{M} \frac{\alpha_{i}^{2} \rho_{i}^{2}}{\alpha_{i}^{2} \tau_{i}+\sigma_{n}^{2}} \\
\text { subject to } \quad \sum_{i=1}^{M} \alpha_{i}^{2} \kappa_{i} \leq \gamma_{\text {total }}
\end{gathered}
$$

where $x>0, y>0, \kappa_{i}>0, \rho_{i} \in \mathbb{R}, \tau_{i}>0, i=1, \ldots, M$ are constants. Then the optimal $\alpha_{i}$ s satisfy

$$
\alpha_{i}^{* 2}=\frac{1}{\tau_{i}}\left(\sqrt{\frac{\rho_{i}^{2} \sigma_{n}^{2}}{\lambda \kappa_{i}}}-\sigma_{n}^{2}\right)^{+} .
$$

Assuming that the sensors are ordered so that

$$
\frac{\rho_{1}^{2}}{\kappa_{1}} \geq \cdots \geq \frac{\rho_{M}^{2}}{\kappa_{M}}
$$

the optimal values of $\alpha_{i}^{2}$ for problem (28) can also be expressed as

$$
\alpha_{i}^{* 2}= \begin{cases}\frac{1}{\tau_{i}}\left(\sqrt{\frac{\rho_{i}^{2} \sigma_{n}^{2}}{\lambda \kappa_{i}}}-\sigma_{n}^{2}\right), & i \leq M_{1} \\ 0, & \text { otherwise }\end{cases}
$$

where

$$
\frac{1}{\sqrt{\lambda}}=\frac{\gamma_{\text {total }}+\sum_{i=1}^{M_{1}} \frac{\kappa_{i}}{\tau_{i}} \sigma_{n}^{2}}{\sum_{i=1}^{M_{1}} \frac{\left|\rho_{i}\right|}{\tau_{i}} \sqrt{\kappa_{i} \sigma_{n}^{2}}}
$$

and the number of sensors which are active, $M_{1}$ (which is again unique), satisfies

$$
\frac{\gamma_{\text {total }}+\sum_{i=1}^{M_{1}} \frac{\kappa_{i}}{\tau_{i}} \sigma_{n}^{2}}{\sum_{i=1}^{M_{1}} \frac{\left|\rho_{i}\right|}{\tau_{i}} \sqrt{\kappa_{i} \sigma_{n}^{2}}} \sqrt{\frac{\rho_{M_{1}}^{2} \sigma_{n}^{2}}{\kappa_{M_{1}}}}-\sigma_{n}^{2}>0
$$

and

$$
\frac{\gamma_{\text {total }}+\sum_{i=1}^{M_{1}+1} \frac{\kappa_{i}}{\tau_{i}} \sigma_{n}^{2}}{\sum_{i=1}^{M_{1}+1} \frac{\left|\rho_{i}\right|}{\tau_{i}} \sqrt{\kappa_{i} \sigma_{n}^{2}}} \sqrt{\frac{\rho_{M_{1}+1}^{2} \sigma_{n}^{2}}{\kappa_{M_{1}+1}}}-\sigma_{n}^{2} \leq 0 .
$$

\section{Remarks}

1) In the orthogonal scheme, the solutions of the optimization problems (26) and (28) take the form (27) and (29), respectively. These expressions are reminiscent of the "water-filling" solutions in wireless communications, where only sensors of sufficiently high-quality measurements are allocated power, while sensors with lower quality measurements are turned off. On the other hand, the solutions for problems (19) and (23) have the form (21) and (24), respectively, which indicates that all sensors get allocated some non-zero power when we perform the optimization. The intuition behind this is that in the multi-access scheme, some "averaging" can be done when measurements are added together, which can reduce the effects of noise and improve performance, while this can't be done in the orthogonal scheme so that turning off low quality sensors will save power.

2) The four optimization problems we consider (problems (19), (23), (26), and (28)) have analytical solutions, and can admit distributed implementations, which may be important in large sensor networks. For problem (19) the fusion center can calculate the values $\lambda$ and $\mu$ and broadcast them to all sensors, and for problem (23) the fusion center can calculate and broadcast the quantity

$$
\left(\sum_{j=1}^{M} \frac{\rho_{j}^{2}}{\left(\kappa_{j}+\gamma_{\text {total }} \tau_{j} / \sigma_{n}^{2}\right)^{2}} \kappa_{j}\right)^{-1}
$$

to all sensors. The sensors can then use these quantities and their local information to compute the optimal $\alpha_{i}$ s; see [23]. For problems (26) and (28), the fusion center can compute and broadcast the quantity $\lambda$ to all sensors, which can then determine their optimal $\alpha_{i}$ s using $\lambda$ and their local information; see [24].

\section{Fading Channels with CSI}

We now consider channel gains that are randomly time varying. In this section we let both the sensors and fusion center have CSI so that the $h_{i, k} \mathrm{~s}$ are known, while Section IVE considers fading channels without CSI. We now also allow the amplification factors $\alpha_{i, k}$ to be time varying.

1) Multi-Access: Recall from (4) that the Kalman filter recursion for the error covariances is

$$
P_{k+1}=\frac{a^{2} P_{k} \bar{r}_{k}}{\bar{c}_{k}^{2} P_{k}+\bar{r}_{k}}+\sigma_{w}^{2}
$$

where $\bar{c}_{k} \equiv \sum_{i=1}^{M} \alpha_{i, k} h_{i, k} c_{i}$ and $\bar{r}_{k} \equiv \sum_{i=1}^{M} \alpha_{i, k}^{2} h_{i, k}^{2} \sigma_{i}^{2}+\sigma_{n}^{2}$.

One way in which we can formulate an optimization problem is to minimize the sum of powers used at each time instant, subject to $P_{k+1 \mid k} \leq D$ at all time instances $k$. That is, for all $k$, we want to solve

$$
\begin{aligned}
& \min \sum_{i=1}^{M} \gamma_{i, k}=\sum_{i=1}^{M} \alpha_{i, k}^{2}\left(\frac{c_{i}^{2} \sigma_{w}^{2}}{1-a^{2}}+\sigma_{i}^{2}\right) \\
& \text { subject to } \quad P_{k+1}=\frac{a^{2} P_{k} \bar{r}_{k}}{\bar{c}_{k}^{2} P_{k}+\bar{r}_{k}}+\sigma_{w}^{2} \leq D .
\end{aligned}
$$

The constraint can be rearranged to be equivalent to

$$
\bar{r}_{k}\left(a^{2} P_{k}+\sigma_{w}^{2}-D\right)+\bar{c}_{k}^{2} P_{k}\left(\sigma_{w}^{2}-D\right) \leq 0
$$

which looks rather similar to (17). In fact, once we've solved the problem (30) at an initial time instance, e.g., $k=1$, then $P_{2}=D$ is satisfied so that further problems become essentially identical to what was solved in Section IVA1. Therefore, the only slight difference is in the initial optimization problem, though this is also covered by the general problem (19). 
Another possible optimization problem is to minimize $P_{k+1 \mid k}$ at each time instant subject to a sum power constraint $\gamma_{\text {total }}$ at each time $k$, i.e.,

$$
\begin{aligned}
& \min P_{k+1}=\frac{a^{2} P_{k} \bar{r}_{k}}{\bar{c}_{k}^{2} P_{k}+\bar{r}_{k}}+\sigma_{w}^{2} \\
& \text { subject to } \sum_{i=1}^{M} \alpha_{i, k}^{2}\left(\frac{c_{i}^{2} \sigma_{w}^{2}}{1-a^{2}}+\sigma_{i}^{2}\right) \leq \gamma_{\text {total }} .
\end{aligned}
$$

As we can rewrite the objective as

$$
\frac{a^{2} P_{k} \bar{r}_{k} / \bar{c}_{k}^{2}}{P_{k}+\bar{r}_{k} / \bar{c}_{k}^{2}}+\sigma_{w}^{2}
$$

it is clear that minimizing the objective function is equivalent to minimizing $\bar{r}_{k} / \bar{c}_{k}^{2}$. So at each time step, we essentially solve the same problem (23) considered in Section IVA2 while updating the value of $P_{k+1}$ every time.

2) Orthogonal Access: Recall from (5) that in the orthogonal scheme, the Kalman filter recursion for the error covariance is

$$
P_{k+1}^{o}=\frac{a^{2} P_{k}^{o}}{1+P_{k}^{o} \overline{\mathbf{C}}_{k}^{o T} \overline{\mathbf{R}}_{k}^{o^{-1}} \overline{\mathbf{C}}_{k}^{o}}+\sigma_{w}^{2} .
$$

If we wish to minimize the sum power while keeping $P_{k+1}^{o} \leq D$ at all time instances, the constraint becomes

$$
\overline{\mathbf{C}}_{k}^{o^{T}} \overline{\mathbf{R}}_{k}^{o^{-1}} \overline{\mathbf{C}}_{k}^{o}=\sum_{i=1}^{M} \frac{\alpha_{i, k}^{2} h_{i, k}^{2} c_{i}^{2}}{\alpha_{i, k}^{2} h_{i, k}^{2} \sigma_{i}^{2}+\sigma_{n}^{2}} \geq \frac{a^{2} P_{k}^{o}+\sigma_{w}^{2}-D}{P_{k}^{o}\left(D-\sigma_{w}^{2}\right)} .
$$

If we wish to minimize $P_{k+1}^{o}$ at each time instance subject to a sum power constraint at all times $k$, then this is the same as maximizing

$$
\overline{\mathbf{C}}_{k}^{o T} \overline{\mathbf{R}}_{k}^{o^{-1}} \overline{\mathbf{C}}_{k}^{o}=\sum_{i=1}^{M} \frac{\alpha_{i, k}^{2} h_{i, k}^{2} c_{i}^{2}}{\alpha_{i, k}^{2} h_{i, k}^{2} \sigma_{i}^{2}+\sigma_{n}^{2}} .
$$

In both cases, the resulting optimization problems which are to be solved at each time instant are variants of problems (26) and (28), and can be handled using the same techniques.

3) Remarks: As discussed in Section IVC, these problems can be solved in a distributed manner, with the fusion center broadcasting some global constants that can then be used by the individual sensors to compute their optimal power allocation. The main issue with running these optimizations at every time step is the cost of obtaining CSI. If the channels don't vary too quickly, one might be able to use the same values for the channel gains over a number of different time steps. However if the channels vary quickly, then estimating the channels at each time step may not be feasible or practical. In this case we propose one possible alternative, which is the use of a linear estimator that depends only on the channel statistics and which is derived in Section IVE.

4) A Dynamic Programming Formulation: The optimization problems we have formulated in this section follow a "greedy" approach where we have constraints that must be satisfied at each time step, which allows us to use the same techniques as in Sections IVA and IVB. An alternative formulation is to consider constraints on the long term averages of the estimation error and transmission powers. For instance, instead of problem (31), one might consider the infinite horizon problem:

$$
\begin{aligned}
& \min \lim _{T \rightarrow \infty} \frac{1}{T} \sum_{k=1}^{T} \mathbb{E}\left[P_{k+1}\right] \\
& \text { subject to } \lim _{T \rightarrow \infty} \frac{1}{T} \sum_{k=1}^{T} \mathbb{E}\left[\sum_{i=1}^{M} \gamma_{i, k}\right] \leq \gamma_{\text {total }}
\end{aligned}
$$

where we wish to determine policies that will minimize the expected error covariance subject to the average sum power being less than a threshold $\gamma_{\text {total }}$. Solving such problems will require dynamic programming techniques, as well as discretization of the optimization variables similar to [46], where optimal quantizers were designed for hidden Markov model (HMM) state estimation over bandwidth constrained channels using a stochastic control approach. This approach is however highly computationally demanding. A thorough study of these problems is beyond the scope of this paper and is currently under investigation.

\section{E. Fading Channels without CSI}

Suppose now that CSI is not available at either the sensors or the fusion center, though channel statistics are available. ${ }^{8}$ The optimal filters in this case will be nonlinear and highly complex, see e.g., [47]. An alternative is to consider the best linear estimator in the MMSE sense based on [37]. In our notation, the situation considered in [37] would be applicable to the model $x_{k+1}=a x_{k}+w_{k}, z_{k}=\alpha_{k} h_{k} c x_{k}+v_{k}$. While this is not quite the same as the situations that we are considering in this paper, these techniques can be suitably extended.

1) Multi-Access Scheme: Since we do not have CSI, we cannot do transmitter beamforming and must return to the full complex model (1). We also restrict $\tilde{\alpha}_{i, k}=\tilde{\alpha}_{i}, \forall k$ to be time invariant. The main difference from [37] is that the innovations are now defined as

$$
\left[\begin{array}{l}
\Re\left[\tilde{z}_{k}\right] \\
\Im\left[\tilde{z}_{k}\right]
\end{array}\right]-\left[\begin{array}{l}
\sum_{i=1}^{M} \mathbb{E}\left[\Re\left[\tilde{\alpha}_{i} \tilde{h}_{i}\right]\right] c_{i} \\
\sum_{i=1}^{M} \mathbb{E}\left[\Im\left[\tilde{\alpha}_{i} \tilde{h}_{i}\right]\right] c_{i}
\end{array}\right] \hat{x}_{k \mid k-1} .
$$

Assuming that the processes $\left\{\tilde{h}_{i, k}\right\}, i=1, \ldots, M$ are IID over time with real and imaginary components independent of each other and $\left\{\tilde{h}_{i, k}\right\}$ independent of $\left\{w_{k}\right\}$ and $\left\{v_{i, k}\right\}, i=1, \ldots, M$, the linear MMSE

\footnotetext{
${ }^{8}$ We note that this can also be used to model the situation where the sensors are not perfectly synchronized [25].
} 
estimator for scalar systems can then be derived following the methods of [37] (also see [48]) as follows:

$$
\begin{aligned}
\hat{x}_{k+1 \mid k}= & a \hat{x}_{k \mid k} \\
P_{k+1 \mid k}= & a^{2} P_{k \mid k} \\
\hat{x}_{k+1 \mid k+1}= & \hat{x}_{k+1 \mid k}+P_{k+1 \mid k} \overline{\overline{\mathbf{C}}}^{T}\left(\overline{\overline{\mathbf{C}}} P_{k+1 \mid k} \overline{\overline{\mathbf{C}}}^{T}+\overline{\overline{\mathbf{R}}}\right)^{-1} \\
& \times\left(\left(\Re\left[\tilde{z}_{k+1}\right], \Im\left[\tilde{z}_{k+1}\right]\right)^{T}-\overline{\overline{\mathbf{C}}} \hat{x}_{k+1 \mid k}\right) \\
P_{k+1 \mid k+1}= & P_{k+1 \mid k}-P_{k+1 \mid k}^{2} \overline{\overline{\mathbf{C}}}^{T}\left(\overline{\overline{\mathbf{C}}} P_{k+1 \mid k} \overline{\overline{\mathbf{C}}}^{T}+\overline{\overline{\mathbf{R}}}\right)^{-1}
\end{aligned}
$$

where

$$
\overline{\overline{\mathbf{C}}} \equiv\left[\sum_{i=1}^{M} \mathbb{E}\left[\Re\left[\tilde{\alpha}_{i} \tilde{h}_{i}\right]\right] c_{i} \sum_{i=1}^{M} \mathbb{E}\left[\Im\left[\tilde{\alpha}_{i} \tilde{h}_{i}\right]\right] c_{i}\right]^{T}
$$

and

$$
\overline{\overline{\mathbf{R}}} \equiv\left[\begin{array}{c}
\left.\sum_{i=1}^{M}\left(\operatorname{Var}\left[\Re\left[\tilde{\alpha}_{i} \tilde{h}_{i}\right]\right] \frac{c_{i}^{2} \sigma_{w}^{2}}{1-a^{2}}+\mathbb{E}\left[\Re^{2}\left[\tilde{\alpha}_{i} \tilde{h}_{i}\right]\right] \sigma_{i}^{2}\right)+\sigma_{n}^{2}\right] \\
\sum_{i=1}^{M} \mathbb{E}\left[\Re\left[\tilde{\alpha}_{i} \tilde{h}_{i}\right]\right] \mathbb{E}\left[\Im\left[\tilde{\alpha}_{i} \tilde{h}_{i}\right]\right] \sigma_{i}^{2}
\end{array}\right.
$$

with $\alpha_{i} \in \mathbb{R}$. Then $S$ simplifies to

$$
S=\frac{\left(\sum_{i=1}^{M} \mathbb{E}\left[\Re\left[\tilde{\alpha}_{i} \tilde{h}_{i}\right]\right] c_{i}\right)^{2}}{\sum_{i=1}^{M}\left(\operatorname{Var}\left[\Re\left[\tilde{\alpha}_{i} \tilde{h}_{i}\right]\right] c_{i}^{2} \frac{\sigma_{w}^{2}}{1-a^{2}}+\mathbb{E}\left[\Re^{2}\left[\tilde{\alpha}_{i} \tilde{h}_{i}\right]\right] \sigma_{i}^{2}\right)+\sigma_{n}^{2}}
$$

where we can find

$$
\begin{aligned}
& \mathbb{E}\left[\Re\left[\tilde{\alpha}_{i} \tilde{h}_{i}\right]\right]=\alpha_{i}\left|\mathbb{E}\left[\tilde{h}_{i}\right]\right| \\
& \operatorname{Var}\left[\Re\left[\tilde{\alpha}_{i} \tilde{h}_{i}\right]\right]=\frac{\alpha_{i}^{2}}{\mid \mathbb{E}\left[\tilde{h}_{i}\right]^{2}}\left(\mathbb{E}^{2}\left[\Re \tilde{h}_{i}\right] \operatorname{Var}\left[\Re \tilde{h}_{i}\right]\right. \\
&\left.+\mathbb{E}^{2}\left[\Im \tilde{h}_{i}\right] \operatorname{Var}\left[\Im \tilde{h}_{i}\right]\right) \\
& \mathbb{E}\left[\Re^{2}\left[\tilde{\alpha}_{i} \tilde{h}_{i}\right]\right]=\frac{\alpha_{i}^{2}}{\mid \mathbb{E}\left[\left.\tilde{h}_{i}\right|^{2}\right.}\left(\mathbb{E}^{2}\left[\Re \tilde{h}_{i}\right] \mathbb{E}\left[\Re^{2} \tilde{h}_{i}\right]\right. \\
&+2 \mathbb{E}^{2}\left[\Re \tilde{h}_{i}\right] \mathbb{E}^{2}\left[\Im \tilde{h}_{i}\right] \\
&\left.+\mathbb{E}^{2}\left[\Im \tilde{h}_{i}\right] \mathbb{E}\left[\Im^{2} \tilde{h}_{i}\right]\right)
\end{aligned}
$$

$$
\left.\begin{array}{c}
\sum_{i=1}^{M} \mathbb{E}\left[\Re\left[\tilde{\alpha}_{i} \tilde{h}_{i}\right]\right] \mathbb{E}\left[\Im\left[\tilde{\alpha}_{i} \tilde{h}_{i}\right]\right] \sigma_{i}^{2} \\
\sum_{i=1}^{M}\left(\operatorname{Var}\left[\Im\left[\tilde{\alpha}_{i} \tilde{h}_{i}\right]\right] \frac{c_{i}^{2} \sigma_{w}^{2}}{1-a^{2}}+\mathbb{E}\left[\Im^{2}\left[\tilde{\alpha}_{i} \tilde{h}_{i}\right]\right] \sigma_{i}^{2}\right)+\sigma_{n}^{2}
\end{array}\right]
$$

using the shorthand $\Re^{2}[X]=(\Re[X])^{2}$ and $\Im^{2}[X]=$ $(\Im[X])^{2}$.

These equations look like the Kalman filter equations but with different $\mathbf{C}$ and $\mathbf{R}$ matrices, so much of our previous analysis will apply. ${ }^{9}$ For instance, since the estimator is not using the instantaneous time-varying channel gains, but only the channel statistics (which are assumed to be constant), there will be a steady-state error covariance given by

$$
P_{\infty}=\frac{\left(a^{2}-1\right)+\sigma_{w}^{2} S+\sqrt{\left(a^{2}-1+\sigma_{w}^{2} S\right)^{2}+4 \sigma_{w}^{2} S}}{2 S}
$$

with $S \equiv \overline{\overline{\mathbf{C}}}^{T} \overline{\overline{\mathbf{R}}}^{-1} \overline{\overline{\mathbf{C}}}$. Note that for circularly symmetric fading channels, e.g., Rayleigh, we have $\overline{\overline{\mathbf{C}}}=\left[\begin{array}{ll}0 & 0\end{array}\right]$, and estimates obtained using this estimator will not be useful. ${ }^{10}$ Thus we now restrict ourselves to non-zero mean fading processes. Motivated by transmitter beamforming in the case with CSI, let us use amplification factors of the form

$$
\tilde{\alpha}_{i}=\alpha_{i} \frac{\left(\mathbb{E}\left[\tilde{h}_{i}\right]\right)^{*}}{\left|\mathbb{E}\left[\tilde{h}_{i}\right]\right|}
$$

\footnotetext{
${ }^{9}$ In fact one can regard it as an "equivalent" linear system (with a stable dynamics and stationary noise processes) along the lines of [48].

${ }^{10}$ Other work where there are difficulties with circularly symmetric fading include [9], [25], [44]. A possible scheme for estimation of IID processes and zero-mean channels which can achieve a $1 / \log M$ scaling has been proposed in [44].
}

using the shorthand $\mathbb{E}^{2}[X]=(\mathbb{E}[X])^{2}, \Re^{2}[X]=(\Re[X])^{2}$ and $\Im^{2}[X]=(\Im[X])^{2}$. If the real and imaginary parts are identically distributed, we have the further simplifications $\operatorname{Var}\left[\Re\left[\tilde{\alpha}_{i} \tilde{h}_{i}\right]\right]=\alpha_{i}^{2} \operatorname{Var}\left[\Re \tilde{h}_{i}\right]$ and $\mathbb{E}\left[\Re^{2}\left[\tilde{\alpha}_{i} \tilde{h}_{i}\right]\right]=\alpha_{i}^{2}\left(\mathbb{E}\left[\Re^{2} \tilde{h}_{i}\right]+\mathbb{E}^{2}\left[\Re \tilde{h}_{i}\right]\right)$.

Power allocation using this suboptimal estimator can then be developed, and the resulting optimization problems (which are omitted for brevity) will be variants of problems (19) and (23). We note however that the optimization problems will only need to be run once since $\overline{\overline{\mathbf{C}}}$ and $\overline{\overline{\mathbf{R}}}$ are time-invariant quantities, rather than at each time instance as in the case with CSI.

Since we have a steady-state error covariance using this estimator, asymptotic behaviour can also be analyzed using the techniques in Sections III. The details are omitted for brevity.

2) Orthogonal Access Scheme: For orthogonal access and no CSI, the equations for the linear MMSE can also be similarly derived and are of the form (32), substituting $\stackrel{\overline{\mathbf{C}}}{\overline{\mathbf{C}}}$ in place of $\overline{\overline{\mathbf{C}}}, \overline{\overline{\mathbf{R}}}^{o}$ in place of $\overline{\overline{\mathbf{R}}}$, etc. We have $\overline{\overline{\mathbf{C}}} \equiv\left[\mathbb{E}\left[\Re\left[\tilde{\alpha}_{1} \tilde{h}_{1}\right]\right] c_{1} \mathbb{E}\left[\Im\left[\tilde{\alpha}_{1} \tilde{h}_{1}\right]\right] c_{1} \ldots\right.$ $\left.\mathbb{E}\left[\Re\left[\tilde{\alpha}_{M} \tilde{h}_{M}\right]\right] c_{M} \mathbb{E}\left[\Im\left[\tilde{\alpha}_{M} \tilde{h}_{M}\right]\right] c_{M}\right]^{T}$ and

$$
\overline{\overline{\mathbf{R}}}^{o} \equiv\left[\begin{array}{ccc}
\overline{\overline{\mathbf{R}}}_{11}^{o} & \cdots & 0 \\
\vdots & \ddots & \vdots \\
0 & \cdots & \overline{\overline{\mathbf{R}}}_{M M}^{o}
\end{array}\right]
$$


with each $\overline{\overline{\mathbf{R}}}_{i i}^{o}$ being a block matrix

$$
\overline{\overline{\mathbf{R}}}_{i i}^{o} \equiv\left[\begin{array}{c}
\operatorname{Var}\left[\Re\left[\tilde{\alpha}_{i} \tilde{h}_{i}\right]\right] c_{i}^{2} \frac{\sigma_{w}^{2}}{1-a^{2}}+\mathbb{E}\left[\Re^{2}\left[\tilde{\alpha}_{i} \tilde{h}_{i}\right]\right] \sigma_{i}^{2}+\sigma_{n}^{2} \\
\mathbb{E}\left[\Re\left[\tilde{\alpha}_{i} \tilde{h}_{i}\right]\right] \mathbb{E}\left[\Im\left[\tilde{\alpha}_{i} \tilde{h}_{i}\right]\right] \sigma_{i}^{2}
\end{array}\right.
$$

$\left.\begin{array}{c}\mathbb{E}\left[\Re\left[\tilde{\alpha}_{i} \tilde{h}_{i}\right]\right] \mathbb{E}\left[\Im\left[\tilde{\alpha}_{i} \tilde{h}_{i}\right]\right] \sigma_{i}^{2} \\ \operatorname{Var}\left[\Im\left[\tilde{\alpha}_{i} \tilde{h}_{i}\right]\right] c_{i}^{2} \frac{\sigma_{w}^{2}}{1-a^{2}}+\mathbb{E}\left[\Im^{2}\left[\tilde{\alpha}_{i} \tilde{h}_{i}\right]\right] \sigma_{i}^{2}+\sigma_{n}^{2}\end{array}\right]$.

There will be a steady-state error covariance given by

$$
P_{\infty}^{o}=\frac{\left(a^{2}-1\right)+\sigma_{w}^{2} S^{o}+\sqrt{\left(a^{2}-1+\sigma_{w}^{2} S^{o}\right)^{2}+4 \sigma_{w}^{2} S^{o}}}{2 S^{o}}
$$

with $S^{o}=\overline{\overline{\mathbf{C}}}^{o^{T}} \overline{\overline{\mathbf{R}}}^{o^{-1}} \overline{\overline{\mathbf{C}}}^{o}$. If we choose $\tilde{\alpha}_{i}=$ $\alpha_{i}\left(\mathbb{E}\left[\tilde{h}_{i}\right]\right)^{*} /\left|\mathbb{E}\left[\tilde{h}_{i}\right]\right|$, then $S^{o}$ can be shown to be

$$
S^{o}=\sum_{i=1}^{M} \frac{\left(\mathbb{E}\left[\Re\left[\tilde{\alpha}_{i} \tilde{h}_{i}\right]\right] c_{i}\right)^{2}}{\left(\operatorname{Var}\left[\Re\left[\tilde{\alpha}_{i} \tilde{h}_{i}\right]\right] c_{i}^{2} \frac{\sigma_{w}^{2}}{1-a^{2}}+\mathbb{E}\left[\Re^{2}\left[\tilde{\alpha}_{i} \tilde{h}_{i}\right]\right] \sigma_{i}^{2}\right)+\sigma_{n}^{2}}
$$

where we also refer to (33) for further simplifications of these quantities.

Asymptotic behaviour and optimal power allocation can also be analyzed using the techniques in Sections III and IVB, respectively, and the details are omitted for brevity.

\section{NUMERICAL STUDIES}

\section{A. Static Channels}

First we show some plots for the asymptotic results of Section III. In Fig. 1(a), we plot $P_{\infty}$ versus $M$ in the multi-access scheme for the symmetric situation with $\alpha_{i}=1 / \sqrt{M}$ and $a=0.8, \sigma_{w}^{2}=1.5$, $\sigma_{n}^{2}=1, c=1, \sigma_{v}^{2}=1, h=0.8$. We compare this with the asymptotic expression

$$
\sigma_{w}^{2}+\frac{a^{2}\left(\sigma_{v}^{2}+\sigma_{n}^{2} / h^{2}\right)}{c^{2}} \frac{1}{M}
$$

from (13). Fig. 1(b) plots the difference between $P_{\infty}-\sigma_{w}^{2}$, and compares this with the term

$$
\frac{a^{2}\left(\sigma_{v}^{2}+\sigma_{n}^{2} / h^{2}\right)}{c^{2}} \frac{1}{M} \text {. }
$$

We can see that $P_{\infty}$ is well approximated by the asymptotic expression even for 20-30 sensors.

In Fig. 2 we plot $P_{\infty}$ versus $M$ in the multi-access scheme with $\alpha_{i}=1 / \sqrt{M}, a=0.9, \sigma_{w}^{2}=1, \sigma_{n}^{2}=1$ and values for $c_{i}, \sigma_{i}^{2}, h_{i}$ chosen from the range $0.5 \leq$ $C_{i} \leq 1,0.5 \leq R_{i} \leq 1,0.5 \leq h_{i} \leq 1$. We also plot the (asymptotic) lower and upper bounds (37) from the proof of Lemma 4,

$$
\sigma_{w}^{2}+\frac{a^{2}\left(h_{\min }^{2} \sigma_{\min }^{2}+\sigma_{n}^{2}\right)}{h_{\max }^{2} c_{\max }^{2}} \frac{1}{M}
$$

and

$$
\sigma_{w}^{2}+\frac{a^{2}\left(h_{\max }^{2} \sigma_{\max }^{2}+\sigma_{n}^{2}\right)}{h_{\min }^{2} c_{\min }^{2}} \frac{1}{M}
$$

It can be seen that $P_{\infty}$ does indeed lie between the two bounds, both of which converge to $\sigma_{w}^{2}$ at the rate $1 / M$.

Next we look at the numerical results for optimal power allocation. In Fig. 3 we compare between using optimal power allocation and equal power allocation for the multi-access scheme. We use $a=$ $0.9, \sigma_{n}^{2}=10^{-9}, \sigma_{w}^{2}=1, c_{i}=1, \forall i$. The sensor noise variances $\sigma_{i}^{2}$ are drawn from a $\chi^{2}(1)$ distribution to model the differences in sensor measurement quality. The channel gains $h_{i}$ are modelled as $d_{i}^{-2}$, with $d_{i}$ representing the distance of sensor $i$ to the fusion center. We use distances uniformly drawn between $20 \mathrm{~m}$ and $100 \mathrm{~m}$. In Fig. 3(a) we keep $D=2$, while in Fig. 3(b) we keep $\gamma_{\text {total }}=10^{-3}$. Each of the data points represent the average over 1000 realisations of the sensor parameters (i.e., $c_{i}, \sigma_{i}^{2}, d_{i}$ ). In Fig. 4 the comparison using the same parameters and parameter distributions is shown for the orthogonal scheme. What can be observed is that as the number of sensors $M$ increases, there is a general trend downwards for both graphs, though optimal power allocation seems to provide more benefits in the orthogonal access scheme than the multi-access scheme.

\section{B. Fading Channels}

In Fig. 5 we compare between the full CSI and no CSI situations for the multi-access scheme using $a=0.9, \sigma_{n}^{2}=10^{-9}, \sigma_{w}^{2}=1, c_{i}=1, \forall i$, and $\sigma_{i}^{2}$ drawn from a $\chi^{2}(1)$ distribution. The complex channel gains $\tilde{h}_{i, k} \mathrm{~s}$ are chosen to be Rician distributed with distance dependence. Specifically, the real and imaginary parts of $\tilde{h}_{i, k}$ are chosen to be distributed as $d_{i}^{-2} \times N\left(\mu_{i}, 1\right)$, with $d_{i}$ uniform between 20 and 100, and $\mu_{i}$ uniform between $1 / 2$ and 1 . In Fig. 5(a) we keep $D=2$, and in Fig. 5(b) we keep $\gamma_{\text {total }}=10^{-3}$. In the full CSI case, the values are averaged over 1000 time steps for each set of sensor parameters (i.e., $c_{i}, \sigma_{i}^{2}, d_{i}$, $\left.\mu_{i}\right)$, and in the no CSI case they are the steady-state values using the linear MMSE estimator (32). The results are then repeated and further averaged over 100 realisations of the sensor parameters. In Fig. 6, we make the same comparison for the orthogonal scheme. We can see in Fig. 5 that for the multi-access scheme the performance loss in the case without CSI is not too great when compared with the case with 


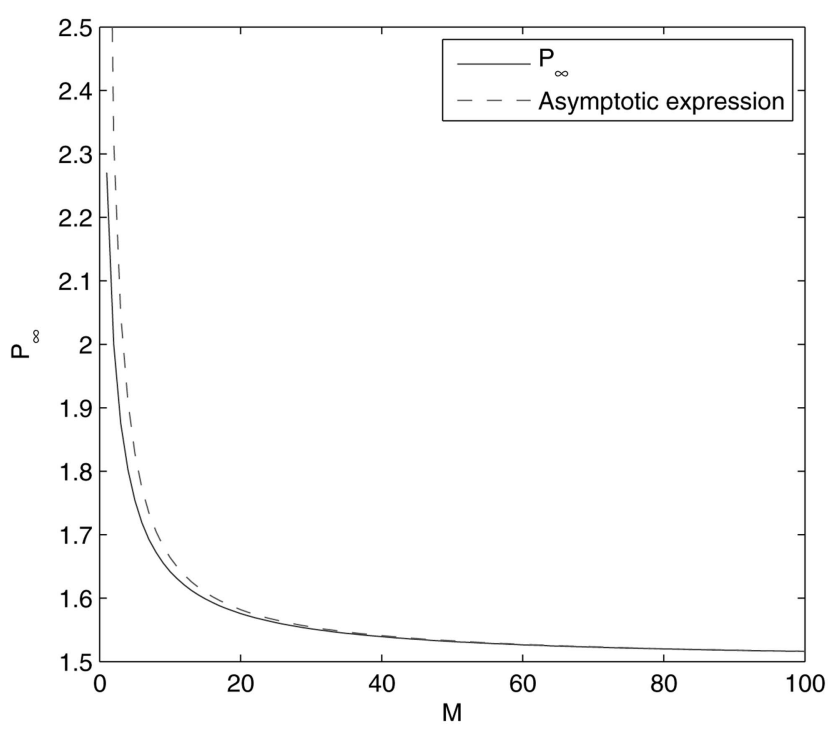

(a)

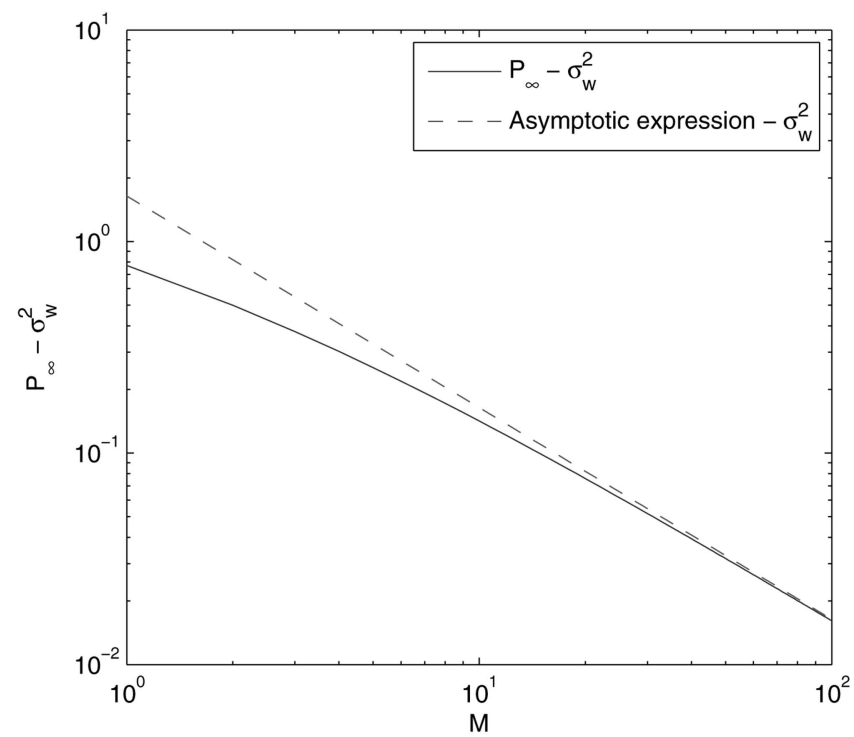

(b)

Fig. 1. Comparison between $P_{\infty}$ and asymptotic expression: multi-access scheme with $\alpha_{i}=1 / \sqrt{M}$.

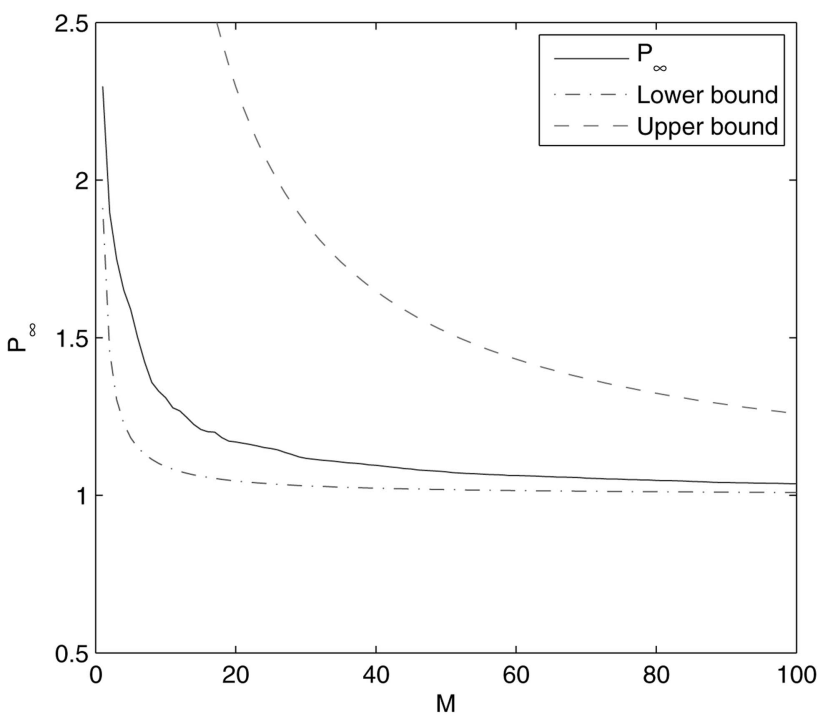

(a)

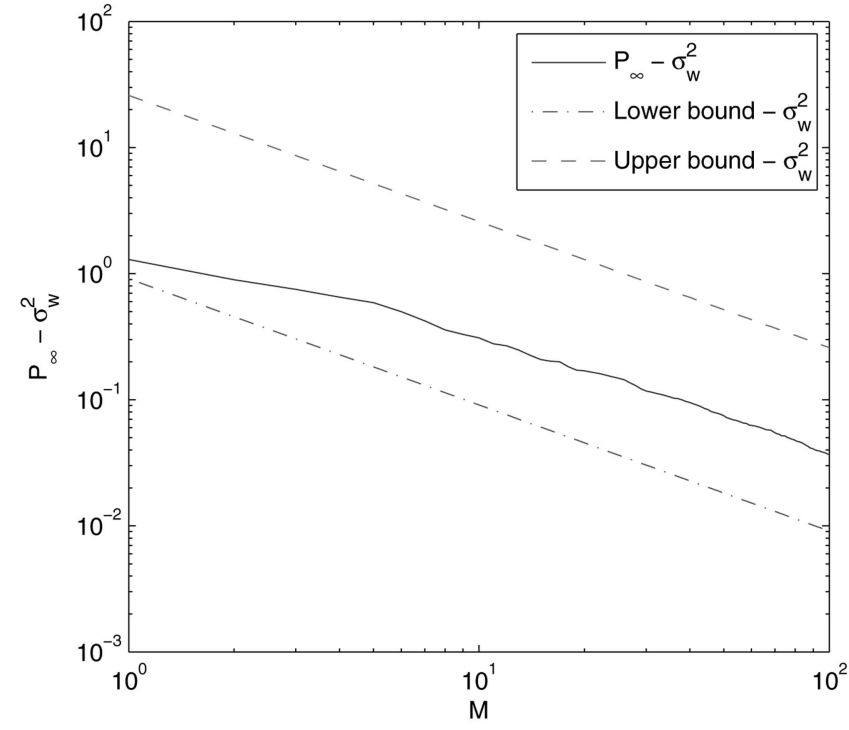

(b)

Fig. 2. $\quad P_{\infty}$ with general parameters and bounds: multi-access scheme with $\alpha_{i}=1 / \sqrt{M}$.

full CSI. Thus even if one has full CSI, but doesn't want to perform power allocation at every time step, using the linear MMSE estimator (32) instead could be an attractive alternative. On the other hand, for the orthogonal scheme in Fig. 6 there is a more significant performance loss in the situation with no CSI.

\section{EXTENSION TO VECTOR STATES AND MIMO}

In Section VIA we formulate a possible extension of our work to vector state linear systems. We outline some of the differences and difficulties that will be encountered when compared with the scalar case. In Section VIB we consider a situation similar to a
MIMO system, where the fusion center has multiple receive antennas (and each sensor operating with a single transmit antenna), and we show how these situations can be expressed as an equivalent vector linear system.

\section{A. Vector States}

We consider a general vector model

$$
\mathbf{x}_{k+1}=\mathbf{A} \mathbf{x}_{k}+\mathbf{w}_{k}
$$

with $\mathbf{x} \in \mathbb{R}^{n}, \mathbf{A} \in \mathbb{R}^{n \times n}$, and $\mathbf{w}_{k} \in \mathbb{R}^{n}$ being Gaussian with zero-mean and covariance matrix $\mathbf{Q}$. For a stable system, all the eigenvalues of the matrix $\mathbf{A}$ will have a magnitude of less than 1 . The $M$ sensors each 


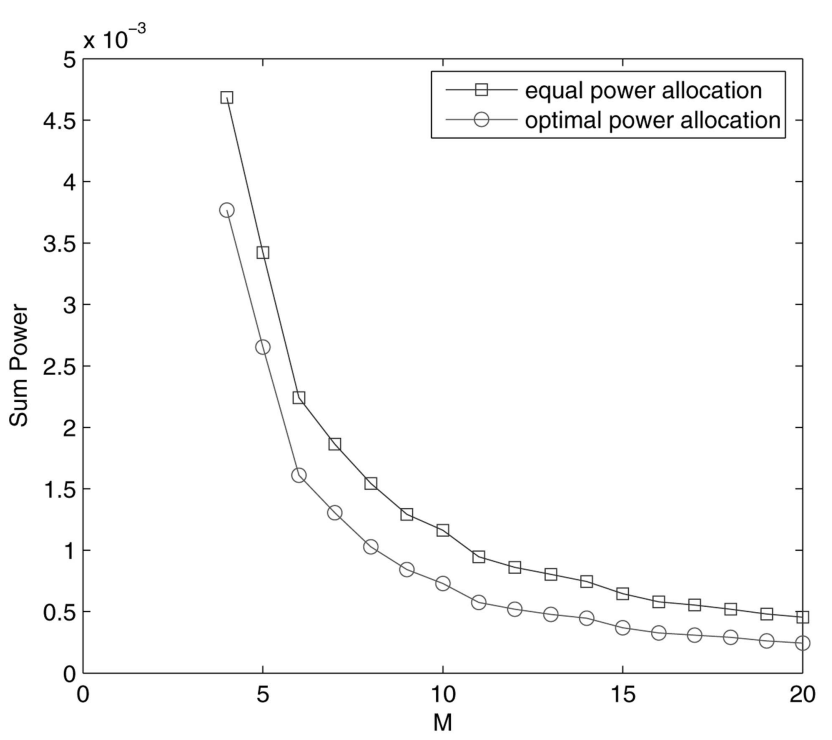

(a)

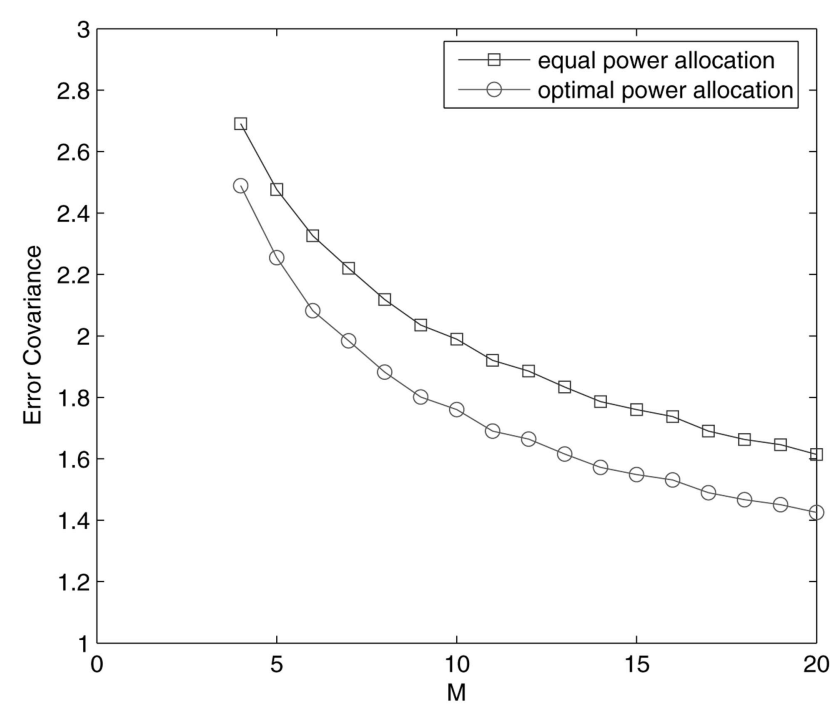

(b)

Fig. 3. Multi-access. Comparison between optimal and equal power allocation schemes with (a) error covariance constraint and (b) sum power constraint.

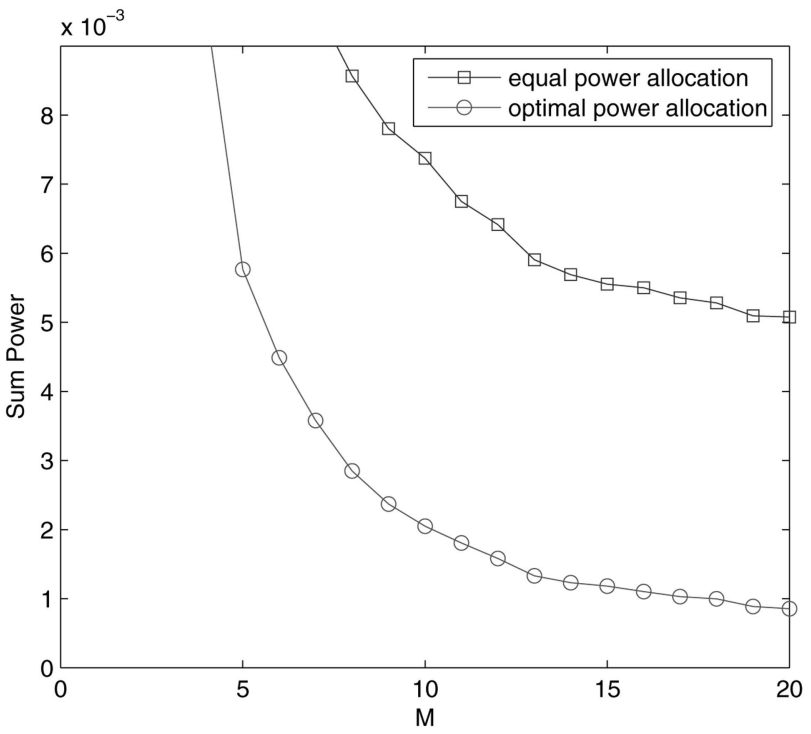

(a)

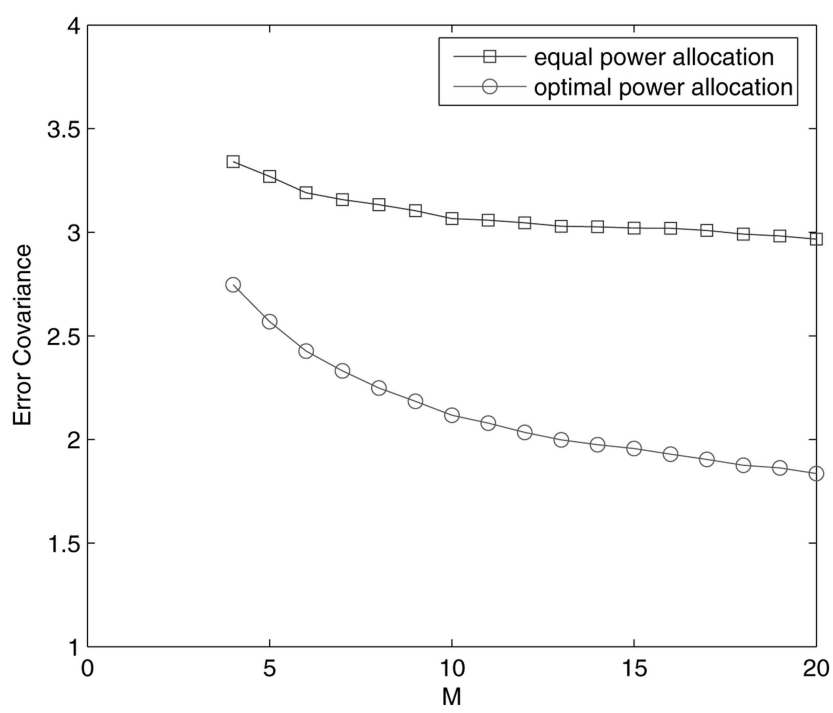

(b)

Fig. 4. Orthogonal access. Comparison between optimal and equal power allocation schemes with (a) error covariance constraint and (b) sum power constraint.

observe

$$
\mathbf{y}_{i, k}=\mathbf{C}_{i} \mathbf{x}_{k}+\mathbf{v}_{i, k}, \quad i=1, \ldots, M
$$

with $\mathbf{y}_{i, k} \in \mathbb{R}^{m}, \mathbf{C}_{i} \in \mathbb{R}^{m \times n}$, and $\mathbf{v}_{i, k} \in \mathbb{R}^{m}$ being Gaussian with zero-mean and covariance matrix $\mathbf{R}_{i}$. We assume that each of the individual components of the measurement vectors $\mathbf{y}_{i, k}$ are amplified and forwarded to a fusion center via separate orthogonal channels. ${ }^{11}$ We consider real channel gains for simplicity.

\footnotetext{
${ }^{11}$ Another possibility is to apply compression on the measured signal $[7,23]$, so that the dimensionality of the signal that the sensor transmits is smaller than the dimension of the measurement vector, but for simplicity we do not consider this here.
}

In the multi-access scheme, the fusion center then receives

$$
\mathbf{z}_{k}=\sum_{i=1}^{M} \mathbf{H}_{i, k} \boldsymbol{\alpha}_{i, k} \mathbf{y}_{i, k}+\mathbf{n}_{k}
$$

where $\boldsymbol{\alpha}_{i, k} \in \mathbb{R}^{m \times m}$ is a matrix of amplification factors, $\mathbf{H}_{i, k} \in \mathbb{R}^{m \times m}$ a matrix of channel gains, and $\mathbf{n}_{k} \in \mathbb{R}^{m}$ is Gaussian with zero-mean and covariance matrix $\mathbf{N}$. We can express the situation as

$$
\mathbf{x}_{k+1}=\mathbf{A} \mathbf{x}_{k}+\mathbf{w}_{k}, \quad \mathbf{z}_{k}=\overline{\mathbf{C}}_{k} \mathbf{x}_{k}+\overline{\mathbf{v}}_{k}
$$

where $\overline{\mathbf{C}}_{k} \equiv \sum_{i=1}^{M} \mathbf{H}_{i, k} \boldsymbol{\alpha}_{i, k} \mathbf{C}_{i}, \overline{\mathbf{v}}_{k} \equiv \sum_{i=1}^{M} \mathbf{H}_{i, k} \boldsymbol{\alpha}_{i, k} \mathbf{v}_{i, k}+$ $\mathbf{n}_{k}$, with $\overline{\mathbf{v}}_{k}$ having covariance matrix $\overline{\mathbf{R}}_{k} \equiv$ $\sum_{i=1}^{M} \mathbf{H}_{i, k} \boldsymbol{\alpha}_{i, k} \mathbf{R}_{i} \boldsymbol{\alpha}_{i, k}^{T} \mathbf{H}_{i, k}^{T}+\mathbf{N}$. The error covariance 


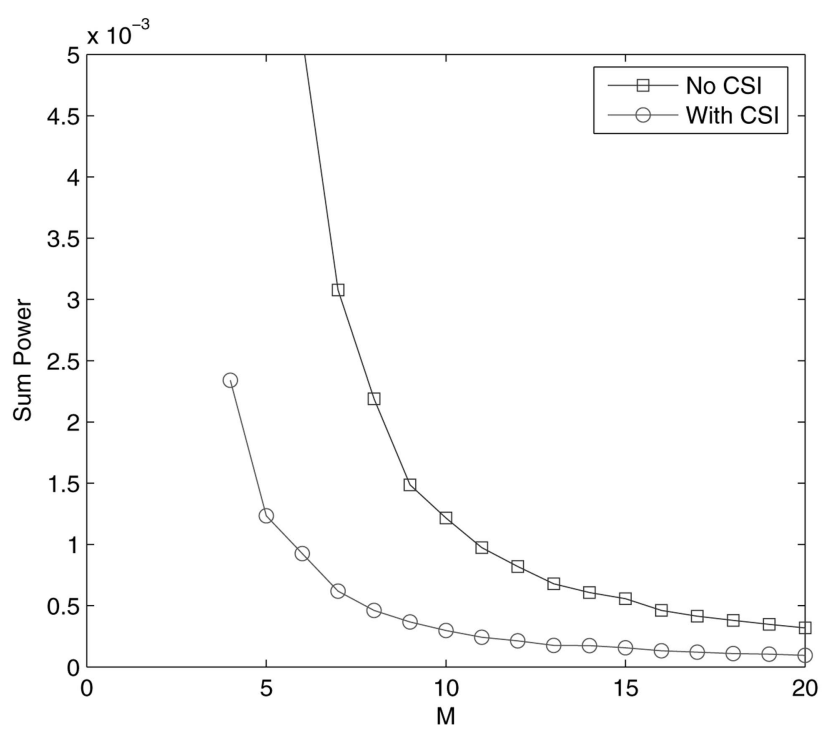

(a)

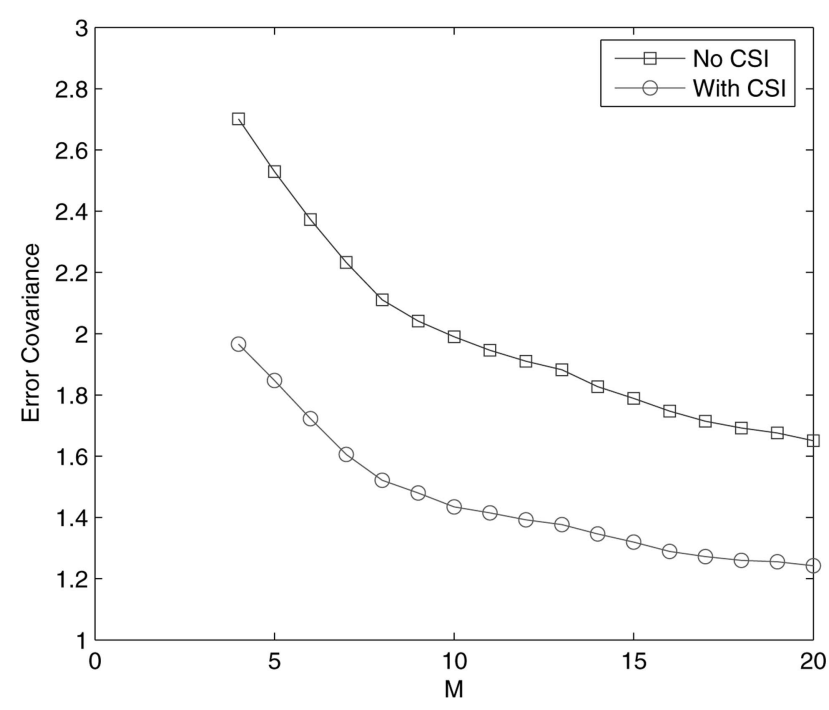

(b)

Fig. 5. Multi-access. Comparison between full CSI and no CSI situations with (a) error covariance constraint and (b) sum power constraint.

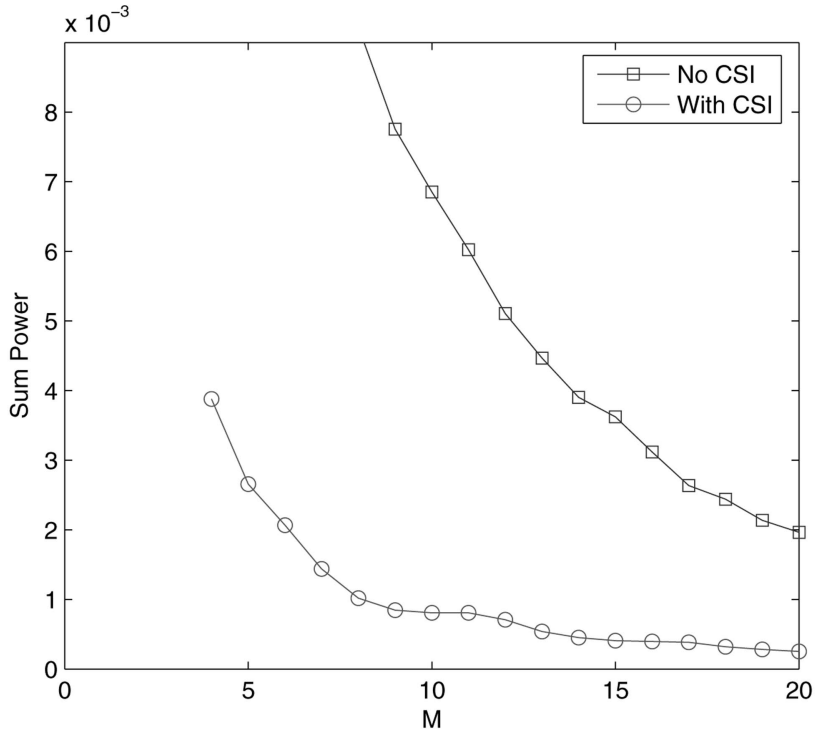

(a)

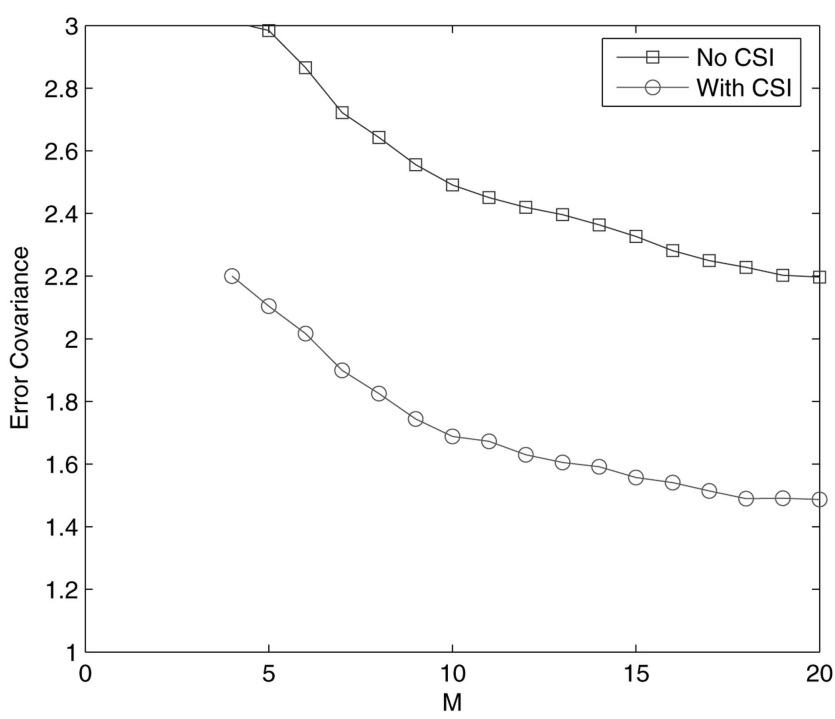

(b)

Fig. 6. Orthogonal access. Comparison between full CSI and no CSI situations with (a) error covariance constraint and (b) sum power constraint.

updates as follows:

$$
\mathbf{P}_{k+1}=\mathbf{A} \mathbf{P}_{k} \mathbf{A}^{T}-\mathbf{A} \mathbf{P}_{k} \overline{\mathbf{C}}_{k}^{T}\left(\overline{\mathbf{C}}_{k} \mathbf{P}_{k} \overline{\mathbf{C}}_{k}^{T}+\overline{\mathbf{R}}_{k}\right)^{-1} \overline{\mathbf{C}}_{k} \mathbf{P}_{k} \mathbf{A}^{T}+\mathbf{Q}
$$

The transmit power of sensor $i$ at time $k$ is

$$
\begin{aligned}
\gamma_{i, k} & =\operatorname{Tr}\left(\boldsymbol{\alpha}_{i, k} \mathbb{E}\left[\mathbf{y}_{k} \mathbf{y}_{k}^{T}\right] \boldsymbol{\alpha}_{i, k}^{T}\right) \\
& =\operatorname{Tr}\left(\boldsymbol{\alpha}_{i, k}\left(\mathbf{C}_{i} \mathbb{E}\left[\mathbf{x}_{k} \mathbf{x}_{k}^{T}\right] \mathbf{C}_{i}^{T}+\mathbf{R}_{i}\right) \boldsymbol{\alpha}_{i, k}^{T}\right)
\end{aligned}
$$

where $\operatorname{Tr}(\cdot)$ denotes the trace, and $\mathbb{E}\left[\mathbf{x}_{k} \mathbf{x}_{k}^{T}\right]$ satisfies (see [41, p. 71])

$$
\mathbb{E}\left[\mathbf{x}_{k} \mathbf{x}_{k}^{T}\right]-\mathbf{A} \mathbb{E}\left[\mathbf{x}_{k} \mathbf{x}_{k}^{T}\right] \mathbf{A}^{T}=\mathbf{Q}
$$

In the static channel case, the steady-state error covariance $\mathbf{P}_{\infty}$ satisfies

$$
\mathbf{P}_{\infty}=\mathbf{A} \mathbf{P}_{\infty} \mathbf{A}^{T}-\mathbf{A} \mathbf{P}_{\infty} \overline{\mathbf{C}}^{T}\left(\overline{\mathbf{C}} \mathbf{P}_{\infty} \overline{\mathbf{C}}^{T}+\overline{\mathbf{R}}\right)^{-1} \overline{\mathbf{C}} \mathbf{P}_{\infty} \mathbf{A}^{T}+\mathbf{Q} .
$$

However, unlike the scalar case where the closed-form expression (7) exists, in the vector case no such formula for $\mathbf{P}_{\infty}$ is available, and thus asymptotic analysis is difficult to develop. For time-varying channels, we can pose similar optimization problems as considered in Section IV. For instance, minimization of the error covariance subject to a sum power constraint can be written 
as

$$
\begin{aligned}
& \min _{\boldsymbol{\alpha}_{1, k}, \ldots, \boldsymbol{\alpha}_{M, k}} \operatorname{Tr}\left(\mathbf{P}_{k+1}\right) \\
& \text { subject to } \\
& \sum_{i=1}^{M}\left(\boldsymbol{\alpha}_{i, k}\left(\mathbf{C}_{i} \mathbb{E}\left[\mathbf{x}_{k} \mathbf{x}_{k}^{T}\right] \mathbf{C}_{i}^{T}+\mathbf{R}_{i}\right) \boldsymbol{\alpha}_{i, k}^{T}\right) \leq \gamma_{\text {total }} .
\end{aligned}
$$

This problem is nonconvex and, unlike the scalar case, does not appear to be able to be reformulated into a convex problem. Similar problems have been considered previously in the context of parameter estimation, and suboptimal solutions were presented using techniques such as deriving bounds on the error covariance [27] and convex relaxation techniques [23].

In the orthogonal-access scheme the fusion center receives

$$
\mathbf{z}_{i, k}=\mathbf{H}_{i, k} \boldsymbol{\alpha}_{i, k} \mathbf{y}_{i, k}+\mathbf{n}_{i, k}, \quad i=1, \ldots, M .
$$

We can express the situation as

$$
\mathbf{x}_{k+1}=\mathbf{A} \mathbf{x}_{k}+\mathbf{w}_{k}, \quad \mathbf{z}_{k}^{o}=\overline{\mathbf{C}}_{k}^{o} \mathbf{x}_{k}+\overline{\mathbf{v}}_{k}^{o}
$$

by defining

$$
\begin{aligned}
\mathbf{z}_{k}^{o} \equiv\left[\begin{array}{c}
\mathbf{z}_{1, k} \\
\vdots \\
\mathbf{z}_{M, k}
\end{array}\right] \\
\overline{\mathbf{C}}_{k}^{o} \equiv\left[\begin{array}{c}
\mathbf{H}_{1, k} \boldsymbol{\alpha}_{1, k} \mathbf{C}_{1} \\
\vdots \\
\mathbf{H}_{M, k} \boldsymbol{\alpha}_{M, k} \mathbf{C}_{M}
\end{array}\right] \\
\overline{\mathbf{v}}_{k}^{o} \equiv\left[\begin{array}{c}
\mathbf{H}_{1, k} \boldsymbol{\alpha}_{1, k} \mathbf{v}_{1, k}+\mathbf{n}_{1, k} \\
\vdots \\
\mathbf{H}_{M, k} \boldsymbol{\alpha}_{M, k} \mathbf{v}_{M, k}+\mathbf{n}_{M, k}
\end{array}\right]
\end{aligned}
$$

with the covariance of $\overline{\mathbf{v}}_{k}^{o}$ being where we have the simplification

$$
\begin{aligned}
\mathbf{C}_{k}^{o^{T}} \overline{\mathbf{R}}_{k}^{o^{-1}} \overline{\mathbf{C}}_{k}^{o^{T}} & \\
& =\sum_{i=1}^{M}\left(\mathbf{H}_{i, k} \boldsymbol{\alpha}_{i, k} \mathbf{C}_{i}\right)^{T}\left(\mathbf{H}_{i, k} \boldsymbol{\alpha}_{i, k} \mathbf{R}_{i} \boldsymbol{\alpha}_{i, k}^{T} \mathbf{H}_{i, k}^{T}+\mathbf{N}\right)^{-1}\left(\mathbf{H}_{i, k} \boldsymbol{\alpha}_{i, k} \mathbf{C}_{i}\right) .
\end{aligned}
$$

Minimization of the error covariance subject to a sum power constraint can be written as

$$
\begin{aligned}
& \min _{\boldsymbol{\alpha}_{1, k}, \ldots, \boldsymbol{\alpha}_{M, k}} \operatorname{Tr}\left(\mathbf{P}_{k+1}^{o}\right) \\
& \text { subject to } \\
& \sum_{i=1}^{M}\left(\boldsymbol{\alpha}_{i, k}\left(\mathbf{C}_{i} \mathbb{E}\left[\mathbf{x}_{k} \mathbf{x}_{k}^{T}\right] \mathbf{C}_{i}^{T}+\mathbf{R}_{i}\right) \boldsymbol{\alpha}_{i, k}^{T}\right) \leq \gamma_{\text {total }} .
\end{aligned}
$$

This problem is nonconvex and also does not appear to be able to be reformulated into a convex problem. In the context of parameter estimation with sensors communicating to a fusion center via orthogonal channels, a similar problem was considered in [49], and was in fact shown to be NP-hard, although suboptimal methods for solving that problem were later studied in [7].

As the techniques involved are quite different from what has currently been presented, a comprehensive study of optimization problems such as (34) and (35) is beyond the scope of this paper.

\section{B. MIMO Situation}

One could also consider a situation resembling the MIMO systems in wireless communications with the different sensors (each with a single transmit antenna) representing the multiple transmitters and multiple receive antennas at the fusion center. It turns out that these situations can be expressed as equivalent vector linear systems. We show how this is done for a simple

$$
\overline{\mathbf{R}}_{k}^{o} \equiv\left[\begin{array}{c}
\mathbf{H}_{1, k} \boldsymbol{\alpha}_{1, k} \mathbf{R}_{1} \boldsymbol{\alpha}_{1, k}^{T} \mathbf{H}_{1, k}^{T}+\mathbf{N} \\
0 \\
\vdots \\
0
\end{array}\right.
$$

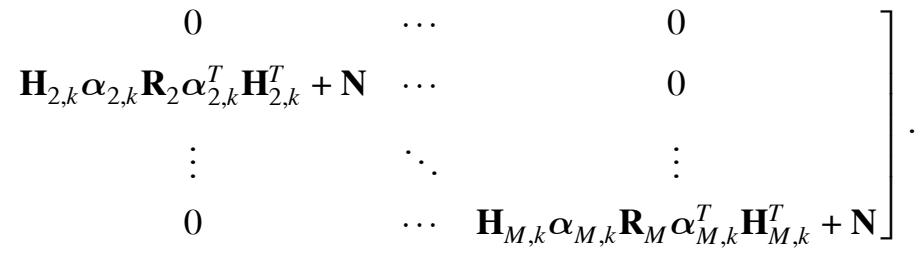

The error covariance updates as follows:

$$
\mathbf{P}_{\mathrm{k}+1}^{\mathrm{o}}=\mathbf{A} \mathbf{P}_{\mathrm{k}}^{\mathrm{o}} \mathbf{A}^{\mathrm{T}}-\mathbf{A} \mathbf{P}_{\mathrm{k}}^{\mathrm{o}} \overline{\mathbf{C}}_{\mathrm{k}}^{\mathrm{o}}\left(\overline{\mathbf{C}}_{\mathrm{k}}^{\mathrm{o}} \mathbf{P}_{\mathrm{k}}^{\mathrm{o}} \overline{\mathbf{C}}_{\mathrm{k}}^{\mathrm{o}^{\mathrm{T}}}+\overline{\mathbf{R}}_{\mathrm{k}}^{\mathrm{o}}\right)^{-1} \overline{\mathbf{C}}_{\mathrm{k}}^{\mathrm{o}} \mathbf{P}_{\mathrm{k}}^{\mathrm{o}} \mathbf{A}^{\mathrm{T}}+\mathbf{Q} .
$$

The term $\overline{\mathbf{C}}_{k}^{o T}\left(\overline{\mathbf{C}}_{k}^{o} \mathbf{P}_{k}^{o} \overline{\mathbf{C}}_{k}^{o T}+\overline{\mathbf{R}}_{k}^{o}\right)^{-1} \overline{\mathbf{C}}_{k}^{o}$ can be rewritten using the matrix inversion lemma as

$$
\begin{aligned}
\overline{\mathbf{C}}_{k}^{o^{T}}\left(\overline{\mathbf{C}}_{k}^{o} \mathbf{P}_{k}^{o} \overline{\mathbf{C}}_{k}^{o^{T}}+\overline{\mathbf{R}}_{k}^{o}\right)^{-1} \overline{\mathbf{C}}_{k}^{o} & \\
= & \mathbf{C}_{k}^{o^{T}} \overline{\mathbf{R}}_{k}^{o^{-1}} \overline{\mathbf{C}}_{k}^{o^{T}}-\mathbf{C}_{k}^{o^{T}} \overline{\mathbf{R}}_{k}^{o^{-1}} \overline{\mathbf{C}}_{k}^{o^{T}}\left(\mathbf{P}_{k}^{o^{-1}}+\mathbf{C}_{k}^{o^{T}} \overline{\mathbf{R}}_{k}^{o^{-1}} \overline{\mathbf{C}}_{k}^{o^{T}}\right)^{-1} \\
& \times \mathbf{C}_{k}^{o^{T}} \overline{\mathbf{R}}_{k}^{o^{-1}} \overline{\mathbf{C}}_{k}^{o^{T}}
\end{aligned}
$$

case. Consider the vector state, scalar measurement system

$$
\mathbf{x}_{k+1}=\mathbf{A} \mathbf{x}_{k}+\mathbf{w}_{k}, \quad y_{i, k}=\mathbf{c}_{i} \mathbf{x}_{k}+v_{i, k}, \quad i=1, \ldots, M
$$

where $\mathbf{c}_{i}, \forall i$ are $1 \times n$ vectors. We look at the orthogonal access scheme, but now with $L$ receive antennas at the fusion center. The fusion center then receives from each sensor

$$
\begin{aligned}
\mathbf{z}_{i, k} & =\left[h_{i, k}^{1} \alpha_{i, k} y_{i, k}+n_{i, k}^{1}, \ldots, h_{i, k}^{L} \alpha_{i, k} y_{i, k}+n_{i, k}^{L}\right]^{T}, \\
i & =1, \ldots, M
\end{aligned}
$$


where $h_{i, k}^{j}$ is the channel gain from the $i$ th sensor to the $j$ th antenna. Defining

$$
\begin{aligned}
\mathbf{z}_{k} \equiv & {\left[\begin{array}{c}
\mathbf{z}_{1, k} \\
\vdots \\
\mathbf{z}_{M, k}
\end{array}\right] } \\
\overline{\mathbf{C}}_{k} \equiv & {\left[h_{1, k}^{1} \alpha_{1, k} \mathbf{c}_{1}^{T}|\cdots| h_{1, k}^{L} \alpha_{1, k} \mathbf{c}_{1}^{T}|\ldots| \cdot\left|h_{M, k}^{1} \alpha_{M, k} \mathbf{c}_{M}^{T}\right|\right.} \\
& \left.\quad \cdots \mid h_{M, k}^{L} \alpha_{M, k} \mathbf{c}_{M}^{T}\right]^{T} \\
\overline{\mathbf{v}}_{k} \equiv & {\left[h_{1, k}^{1} \alpha_{1, k} v_{1, k}+n_{1, k}^{1}, \ldots, h_{1, k}^{L} \alpha_{1, k} v_{1, k}+n_{1, k}^{L}, \ldots, \ldots,\right.} \\
& \left.h_{M, k}^{1} \alpha_{M, k} v_{m, k}+n_{M, k}^{1}, \ldots, h_{M, k}^{L} \alpha_{m, k} v_{m, k}+n_{M, k}^{L}\right]^{T}
\end{aligned}
$$

we may then write the situation as the vector system:

$$
\mathbf{x}_{k+1}=\mathbf{A} \mathbf{x}_{k}+\mathbf{w}_{k}, \quad \mathbf{z}_{k}=\overline{\mathbf{C}}_{k} \mathbf{x}_{k}+\overline{\mathbf{v}}_{k} .
$$

Other variations of the MIMO setup, e.g., vector sensor measurements, can be similarly transformed into equivalent vector linear systems. Note that for scalar state and scalar measurements per sensor, one could use similar techniques to Section IIB for problem formulation and those of Sections IVB and IVD for the optimal power allocation results. However, as described in Section VIA, difficulties in analyzing general vector systems still remain.

\section{CONCLUSION}

This paper has investigated the use of analog amplify and forwarding in the distributed estimation of stable scalar linear systems. We have shown a $1 / M$ scaling behaviour of the error covariance in a number of different situations and formulated and solved some optimal power allocation problems for both static and fading channels. We have also outlined extensions to vector linear systems and MIMO systems. Further study of these extensions and related problems will form the topics of future investigations.

\section{APPENDIX}

A. Proof of Lemma 1

Rewrite (8) as

$$
\begin{aligned}
P_{\infty}= & \frac{\left(a^{2}-1\right)}{2} \frac{1}{S}+\frac{\sigma_{w}^{2}}{2} \\
& +\sqrt{\frac{\left(a^{2}-1\right)^{2}}{4} \frac{1}{S^{2}}+\frac{\left(a^{2}+1\right) \sigma_{w}^{2}}{2} \frac{1}{S}+\frac{\sigma_{w}^{4}}{4} .}
\end{aligned}
$$

Taking the derivative with respect to $S$ we get

$$
\frac{d P_{\infty}}{d S}=-\frac{a^{2}-1}{2} \frac{1}{S^{2}}-\frac{\left(a^{2}-1\right)^{2} \frac{1}{S^{3}}+\left(a^{2}+1\right) \sigma_{w}^{2} \frac{1}{S^{2}}}{4 \sqrt{\frac{\left(a^{2}-1\right)^{2}}{4} \frac{1}{S^{2}}+\frac{\left(a^{2}+1\right) \sigma_{w}^{2}}{2} \frac{1}{S}+\frac{\sigma_{w}^{4}}{4}}} .
$$

To show that $d P_{\infty} / d S \leq 0$, it is sufficient to show that

$$
\begin{gathered}
\left(\frac{\left(a^{2}-1\right)^{2} \frac{1}{S^{3}}+\left(a^{2}+1\right) \sigma_{w}^{2} \frac{1}{S^{2}}}{4 \sqrt{\frac{\left(a^{2}-1\right)^{2}}{4}} \frac{1}{S^{2}}+\frac{\left(a^{2}+1\right) \sigma_{w}^{2}}{2} \frac{1}{S}+\frac{\sigma_{w}^{4}}{4}}\right)^{2} \\
\geq\left(\frac{a^{2}-1}{2} \frac{1}{S^{2}}\right)^{2} .
\end{gathered}
$$

Expanding and rearranging, this is equivalent to

$$
\begin{gathered}
\left(a^{2}-1\right)^{4} \frac{1}{S^{6}}+2\left(a^{2}-1\right)^{2}\left(a^{2}+1\right) \sigma_{w}^{2} \frac{1}{S^{5}}+\left(a^{2}+1\right)^{2} \sigma_{w}^{4} \frac{1}{S^{4}} \\
\geq\left(a^{2}-1\right)^{4} \frac{1}{S^{6}}+2\left(a^{2}-1\right)^{2}\left(a^{2}+1\right) \sigma_{w}^{2} \frac{1}{S^{5}} \\
+\left(a^{2}-1\right)^{2} \sigma_{w}^{4} \frac{1}{S^{4}}
\end{gathered}
$$

or $\left(a^{2}+1\right)^{2} \sigma_{w}^{4} \geq\left(a^{2}-1\right)^{2} \sigma_{w}^{4}$, which is certainly true.

\section{B. Proof of Lemma 3}

We first substitute the simplified expressions for $\bar{c}$ and $\bar{r}$ into (7):

$$
P_{\infty}=\frac{\left(a^{2}-1\right)\left(M h^{2} \sigma_{v}^{2}+\sigma_{n}^{2}\right)+M^{2} h^{2} c^{2} \sigma_{w}^{2}}{2 M^{2} h^{2} c^{2}}+\frac{\sqrt{\left(\left(a^{2}-1\right)\left(M h^{2} \sigma_{v}^{2}+\sigma_{n}^{2}\right)+M^{2} h^{2} c^{2} \sigma_{w}^{2}\right)^{2}+4 M^{2} h^{2} c^{2} \sigma_{w}^{2}\left(M h^{2} \sigma_{v}^{2}+\sigma_{n}^{2}\right)}}{2 M^{2} h^{2} c^{2}} .
$$

Regarded as a function of $M$, we are interested in the behaviour of $P_{\infty}$ as $M \rightarrow \infty$. Now

$$
\begin{aligned}
& \sqrt{\left(\left(a^{2}-1\right)\left(M h^{2} \sigma_{v}^{2}+\sigma_{n}^{2}\right)+M^{2} h^{2} c^{2} \sigma_{w}^{2}\right)^{2}+4 M^{2} h^{2} c^{2} \sigma_{w}^{2}\left(M h^{2} \sigma_{v}^{2}+\sigma_{n}^{2}\right)} \\
& =\left(h^{4} c^{4} \sigma_{w}^{4} M^{4}+2\left(a^{2}-1\right) \sigma_{v}^{2} h^{4} c^{2} \sigma_{w}^{2} M^{3}+4 h^{4} c^{2} \sigma_{w}^{2} \sigma_{v}^{2} M^{3}+O\left(M^{2}\right)\right)^{1 / 2} \\
& =h^{2} c^{2} \sigma_{w}^{2} M^{2}\left(1+\frac{2\left(a^{2}+1\right) \sigma_{v}^{2}}{c^{2} \sigma_{w}^{2} M}+O\left(\frac{1}{M^{2}}\right)\right)^{1 / 2} \\
& =h^{2} c^{2} \sigma_{w}^{2} M^{2}\left(1+\frac{1}{2} \frac{2\left(a^{2}+1\right) \sigma_{v}^{2}}{c^{2} \sigma_{w}^{2} M}+O\left(\frac{1}{M^{2}}\right)\right) \\
& =h^{2} c^{2} \sigma_{w}^{2} M^{2}+\left(a^{2}+1\right) h^{2} \sigma_{v}^{2} M+O(1)
\end{aligned}
$$


where we have used the expansion $(1+x)^{1 / 2}=1+$ $x / 2+O\left(x^{2}\right)$ for $|x|<1[50$, p. 15], which is valid when $M$ is sufficiently large. Hence

$$
P_{\infty}=\sigma_{w}^{2}+\frac{a^{2} \sigma_{v}^{2}}{c^{2}} \frac{1}{M}+O\left(\frac{1}{M^{2}}\right) .
$$

\section{Proof of Lemma 4}

We first prove the statements for the multi-access scheme. We have $M h_{\min } c_{\text {min }} \leq \sum_{i=1}^{M} h_{i} c_{i} \leq M h_{\max } c_{\max }$ and $M h_{\min }^{2} \sigma_{\min }^{2} \leq \sum_{i=1}^{M} h_{i}^{2} \sigma_{i}^{2} \leq M h_{\max }^{2} \sigma_{\max }^{2}$. Recall from Lemma 1 that $P_{\infty}$ is a decreasing function of $S=\bar{c}^{2} / \bar{r}$. If we choose $\alpha_{i} \in\{+1,-1\}$ such that $\alpha_{i} c_{i}$ is positive for all $i$, we have

$$
\frac{M h_{\min }^{2} \sigma_{\min }^{2}+\sigma_{n}^{2}}{M^{2} h_{\max }^{2} c_{\max }^{2}} \leq \frac{\bar{r}}{\bar{c}^{2}} \leq \frac{M h_{\max }^{2} \sigma_{\max }^{2}+\sigma_{n}^{2}}{M^{2} h_{\min }^{2} c_{\min }^{2}}
$$

and by a similar calculation to (36) we can show that as $M \rightarrow \infty$,

$$
\begin{aligned}
\sigma_{w}^{2}+ & \frac{a^{2} h_{\min }^{2} \sigma_{\min }^{2}}{h_{\max }^{2} c_{\max }^{2}} \frac{1}{M}+O\left(\frac{1}{M^{2}}\right) \\
& \leq P_{\infty} \leq \sigma_{w}^{2}+\frac{a^{2} h_{\max }^{2} \sigma_{\max }^{2}}{h_{\min }^{2} c_{\min }^{2}} \frac{1}{M}+O\left(\frac{1}{M^{2}}\right) .
\end{aligned}
$$

If instead we choose $\alpha_{i} \in\{1 / \sqrt{M},-1 / \sqrt{M}\}$ such that $\alpha_{i} c_{i}$ is positive for all $i$, we can similarly show that as $M \rightarrow \infty$,

$$
\begin{aligned}
\sigma_{w}^{2}+ & \frac{a^{2}\left(h_{\min }^{2} \sigma_{\min }^{2}+\sigma_{n}^{2}\right)}{h_{\max }^{2} c_{\max }^{2}} \frac{1}{M}+O\left(\frac{1}{M^{2}}\right) \leq P_{\infty} \\
& \leq \sigma_{w}^{2}+\frac{a^{2}\left(h_{\max }^{2} \sigma_{\max }^{2}+\sigma_{n}^{2}\right)}{h_{\min }^{2} c_{\min }^{2}} \frac{1}{M}+O\left(\frac{1}{M^{2}}\right) .
\end{aligned}
$$

In either case, as the upper and lower bounds both converge to $\sigma_{w}^{2}$ at a rate of $1 / M, P_{\infty}$ itself will also do so.

For the orthogonal scheme, a similar argument to the above shows that choosing $\alpha_{i} \in\{+1,-1\}$ gives convergence of $P_{\infty}^{o}$ to $\sigma_{w}^{2}$ at the rate $1 / M$ for general parameters.

To show that $P_{\infty}^{o}$ in general does not converge to a limit as $M \rightarrow \infty$, when using the scaling $1 / \sqrt{M}$ in the orthogonal scheme, consider the following example. Suppose there are two distinct sets of "symmetric" parameters with behaviour as in (14), such that if all the sensors had the first set of parameters, the error covariance would converge to $P_{\infty, 1}^{o}$, and if all the sensors had the second set of parameters, the error covariance would converge to $P_{\infty, 2}^{o}$, with $P_{\infty, 2}^{o} \neq P_{\infty, 1}^{o}$. Then let the first $M_{1}$ sensors have the first set of parameters, the next $M_{2}$ (with $M_{2} \gg M_{1}$ ) sensors the second set, the next $M_{3}$ (with $M_{3} \gg M_{2}$ ) sensors the first set, the next $M_{4}$ (with $M_{4} \gg M_{3}$ ) sensors the second set, etc.... Then $P_{\infty}^{o}$ will alternate between approaching $P_{\infty, 1}^{o}$ and $P_{\infty, 2}^{o}$ and will not converge to a limit as $M \rightarrow \infty$.

\section{Proof of Lemma 5}

With the multi-access scheme and the allocation (15), by defining

$$
\alpha_{\max }^{2}=\frac{\gamma\left(1-a^{2}\right)}{c_{\min }^{2} \sigma_{w}^{2}+\sigma_{\min }^{2}\left(1-a^{2}\right)}
$$

and

$$
\alpha_{\min }^{2}=\frac{\gamma\left(1-a^{2}\right)}{c_{\max }^{2} \sigma_{w}^{2}+\sigma_{\max }^{2}\left(1-a^{2}\right)}
$$

we can show, similar to the proof of Lemma 4 , that

$$
\frac{M \alpha_{\min }^{2} h_{\min }^{2} \sigma_{\min }^{2}+\sigma_{n}^{2}}{M^{2} \alpha_{\max }^{2} h_{\max }^{2} c_{\max }^{2}} \leq \frac{\bar{r}}{\bar{c}^{2}} \leq \frac{M \alpha_{\max }^{2} h_{\max }^{2} \sigma_{\max }^{2}+\sigma_{n}^{2}}{M^{2} \alpha_{\min }^{2} h_{\min }^{2} c_{\min }^{2}} .
$$

Hence as $M \rightarrow \infty$ we have

$$
\begin{aligned}
\sigma_{w}^{2}+ & \frac{a^{2} \alpha_{\min }^{2} h_{\min }^{2} \sigma_{\min }^{2}}{\alpha_{\max }^{2} h_{\max }^{2} c_{\max }^{2}} \frac{1}{M}+O\left(\frac{1}{M^{2}}\right) \\
& \leq P_{\infty} \leq \sigma_{w}^{2}+\frac{a^{2} \alpha_{\max }^{2} h_{\max }^{2} \sigma_{\max }^{2}}{\alpha_{\min }^{2} h_{\min }^{2} c_{\min }^{2}} \frac{1}{M}+O\left(\frac{1}{M^{2}}\right) .
\end{aligned}
$$

The other cases can be treated similarly as in the proof of Lemma 4.

\section{REFERENCES}

[1] Chamberland, J-F. and Veeravalli, V. V.

Decentralized detection in sensor networks. IEEE Transactions on Signal Processing, 51, 2 (Feb. 2003), 407-416.

[2] Rago, C., Willett, P., and Bar-Shalom, Y.

Censoring sensors: A low-communication-rate scheme for distributed detection.

IEEE Transactions on Aerospace and Electronic Systems, 32, 2 (Apr. 1996), 554-568.

[3] Chen, B., Jiang, R., Kasetkasem, T., and Varshney, P. K. Channel aware decision fusion in wireless sensor networks.

IEEE Transactions on Signal Processing, 52, 12 (Dec. 2004), 3454-3458.

[4] Jiang, R. and Chen, B.

Fusion of censored decisions in wireless sensor networks. IEEE Transactions on Wireless Communications, 4, 6 (Nov. 2005), 2668-2673.

[5] Ribeiro, A. and Giannakis, G. B.

Bandwidth-constrained distributed estimation for wireless sensor networks, Part I: Gaussian case.

IEEE Transactions on Signal Processing, 54, 3 (Mar. 2006), 1131-1143.

[6] Xiao, J-J., Cui, S., Luo, Z-Q., and Goldsmith, A. J. Power scheduling of universal decentralized estimation in sensor networks.

IEEE Transactions on Signal Processing, 54, 2 (Feb. 2006), 413-422.

[7] Schizas, I. D., Giannakis, G. B., and Luo, Z-Q.

Distributed estimation using reduced-dimensionality sensor observations.

IEEE Transactions on Signal Processing, 55, 8 (Aug. 2007), 4284-4299. 
[8] Wu, J-Y., Huang, Q-Z., and Lee, T-S.

Minimal energy decentralized estimation via exploiting the statistical knowledge of sensor noise variance. IEEE Transactions on Signal Processing, 56, 5 (May 2008), 2171-2176.

[9] Mergen, G. and Tong, L.

Type based estimation over multiaccess channels. IEEE Transactions on Signal Processing, 54, 2 (Feb. 2006), 613-626.

[10] Mergen, G., Naware, V., and Tong, L.

Asymptotic detection performance of type-based multiple access over multiaccess fading channels.

IEEE Transactions on Signal Processing, 55, 3 (Mar. 2007), 1081-1092.

[11] Liu, K. and Sayeed, A. M.

Type-based decentralized detection in wireless sensor networks.

IEEE Transactions on Signal Processing, 55, 5 (May 2007), 1899-1910.

[12] Nowak, R., Mitra, U., and Willett, R.

Estimating inhomogenous fields using wireless sensor networks.

IEEE Journal on Selected Areas in Communication, 22, 6 (Aug. 2004), 999-1006.

[13] Wang, Y., Ishwar, P., and Saligrama, V.

One-bit distributed sensing and coding for field estimation in sensor networks.

IEEE Transactions on Signal Processing, 56, 9 (Sept. 2008), 4433-4445.

[14] Zhang, H., Moura, J. M. F., and Krogh, B.

Dynamic field estimation using wireless sensor networks:

Tradeoffs between estimation error and communication

cost.

IEEE Transactions on Signal Processing, 57, 6 (June 2009), 2383-2395.

[15] Gastpar, M. and Vetterli, M.

Source-channel communication in sensor networks. Springer Lecture Notes in Computer Science, 2634 (Apr. 2003), 162-177.

[16] Li, W. and Dai, H.

Distributed detection in wireless sensor networks using a multiple access channel.

IEEE Transactions on Signal Processing, 55, 3 (Mar. 2007), 822-833.

[17] Zhang, X., Poor, H. V., and Chiang, M.

Optimal power allocation for distributed detection over MIMO channels in wireless sensor networks.

IEEE Transactions on Signal Processing, 56, 9 (Sept. 2008), 4124-4140.

[18] Goblick, T. J.

Theoretical limitations on the transmission of data from analog sources.

IEEE Transactions on Information Theory, 11, 4 (Oct. 1965), 558-567.

[19] Gastpar, M., Rimoldi, B., and Vetterli, M.

To code, or not to code: Lossy source-channel communication revisited.

IEEE Transactions on Information Theory, 49, 5 (May 2003), 1147-1158.

[20] Viswanathan, H. and Berger, T.

The quadratic Gaussian CEO problem.

IEEE Transactions on Information Theory, 43, 5 (Sept. 1997), 1549-1559.

[21] Ishwar, P., Puri, R., Ramchandran, K., and Pradhan, S. S. On rate-constrained distributed estimation in unreliable sensor networks.

IEEE Journal on Selected Areas in Communication, 23, 4 (Apr. 2005), 765-775.
[22] Gastpar, M.

Uncoded transmission is exactly optimal for a simple

Gaussian sensor network.

IEEE Transactions on Information Theory, 54, 11 (Nov. 2008), 5247-5251.

[23] Xiao, J-J., Cui, S., Luo, Z-Q., and Goldsmith, A. J. Linear coherent decentralized estimation.

IEEE Transactions on Signal Processing, 56, 2 (Feb. 2008), 757-770.

[24] Cui, S., Xiao, J-J., Goldsmith, A., Luo, Z-Q., and

Poor, H. V.

Estimation diversity and energy efficiency in distributed sensing.

IEEE Transactions on Signal Processing, 55, 9 (Sept. 2007), 4683-4695.

[25] Gastpar, M. and Vetterli, M.

Power, spatio-temporal bandwidth, and distortion in large sensor networks.

IEEE Journal on Selected Areas in Communications, 23, 4

(Apr. 2005), 745-754.

[26] Bahceci, I. and Khandani, A. K.

Linear estimation of correlated data in wireless sensor networks with optimum power allocation and analog modulation.

IEEE Transactions on Communications, 56, 7 (July 2008), 1146-1156.

[27] Fang, J. and Li, H.

Power constrained distributed estimation with correlated sensor data.

IEEE Transactions on Signal Processing, 57, 8 (Aug. 2009), 3292-3297.

[28] Thatte, G. and Mitra, U.

Sensor selection and power allocation for distributed estimation in sensor networks: Beyond the star topology. IEEE Transactions on Signal Processing, 56, 7 (July 2008), 2649-2661.

[29] Hong, Y-W. P., Lei, K-U., and Chi, C-Y.

Channel-aware random access control for distributed estimation in sensor networks.

IEEE Transactions on Signal Processing, 56, 7 (July 2008), 2967-2980.

[30] Şenol, H. and Tepedelenlioğlu, C.

Performance of distributed estimation over unknown parallel fading channels.

IEEE Transactions on Signal Processing, 56, 12 (Dec. 2008), 6057-6068.

[31] Zhang, K. and Li, X. R.

Optimal sensor data quantization for best linear unbiased estimation fusion.

In Proceedings of the IEEE Conference on Decision and Control, Atlantis, Bahamas, Dec. 2004, 2656-2661.

[32] Ribeiro, A., Giannakis, G. B., and Roumeliotis, S. I.

SOI-KF: Distributed Kalman filtering with low-cost communications using the sign of innovations.

IEEE Transactions on Signal Processing, 54, 12 (Dec. 2006), 4782-4795.

[33] Willner, D., Chang, C. B., and Dunn, K. P.

Kalman filter algorithms for a multi-sensor system.

In Proceedings of the IEEE Conference on Decision and Control, Clearwater, FL, Dec. 1976, 570-574.

[34] Hashemipour, H. R., Roy, S., and Laub, A. J.

Decentralized structures for parallel Kalman filtering.

IEEE Transactions on Automatic Control, 33, 1 (Jan. 1988), 88-94.

[35] Rao, B. S. and Durrant-Whyte, H. F.

Fully decentralised algorithm for multisensor Kalman filtering.

IEE Proceedings-D, 138, 5 (Sept. 1991), 413-420. 
[36] Nahi, N. E.

Optimal recursive estimation with uncertain observation. IEEE Transactions on Information Theory, 15, 4 (July 1969), 457-462.

[37] Rajasekaran, P. K., Satyanarayana, N., and Srinath, M. D. Optimum linear estimation of stochastic signals in the presence of multiplicative noise.

IEEE Transactions on Aerospace and Electronic Systems, AES-7, 3 (May 1971), 462-468.

[38] Hadidi, M. T. and Schwartz, S. C.

Linear recursive state estimators under uncertain observations.

IEEE Transactions on Automatic Control, 24, 6 (Dec. 1979), 944-948.

[39] Mudumbai, R., Barriac, G., and Madhow, U.

On the feasibility of distributed beamforming in wireless networks.

IEEE Transactions on Wireless Communications, 6, 5 (May 2007), 1754-1763.

[40] Bucklew, J. A. and Sethares, W. A.

Convergence of a class of decentralized beamforming algorithms.

IEEE Transactions on Signal Processing, 56, 6 (June 2008), 2280-2288.

[41] Aderson, B. D. O. and Moore, J. B.

Optimal Filtering.

Upper Saddle River, NJ: Prentice-Hall, 1979.

[42] Hardy, G. H., Littlewood, J. E., and Pólya, G.

Inequalities (2nd ed.).

London: Cambridge, 1952.

[43] Gan, Q. and Harris, C. J.

Comparison of two measurement fusion methods for

Kalman-filter-based multisensor data fusion.

IEEE Transactions on Aerospace and Electronic Systems,

37, 1 (Jan. 2001), 273-280.
[44] Liu, K., El Gamal, H., and Sayeed, A. M.

Decentralized inference over multiple-access channels. IEEE Transactions on Signal Processing, 5, 7 (July 2007), 3445-3455.

[45] Berger, $M$.

Geometry II.

Berlin: Springer, 1987.

[46] Ghasemi, N. and Dey, S.

Power-efficient dynamic quantization for multisensor HMM state estimation over fading channels.

In Proceedings of the International Symposium on Communications Control and Signal Processing (ISCCSP), Malta, Mar. 2008, 1553-1558.

[47] Jaffer, A. G. and Gupta, S. C.

Recursive Bayesian estimation with uncertain observations.

IEEE Transactions on Information Theory, 17, 5 (Sept. 1971), 614-616.

[48] Tugnait, J. K.

Stability of optimum linear estimators of stochastic signals in white multiplicative noise.

IEEE Transactions on Automatic Control, AC-26, 3 (June 1981), 757-761.

[49] Luo, Z-Q., Giannakis, G. B., and Zhang, S.

Optimal linear decentralized estimation in a bandwidth constrained sensor network.

In Proceedings of the IEEE International Symposium on Information Theory, Adelaide, Australia, Sept. 2005, $1441-1445$

[50] Abramowitz, M. and Stegun, I. A.

Handbook of Mathematical Functions.

New York: Dover, 1965. 

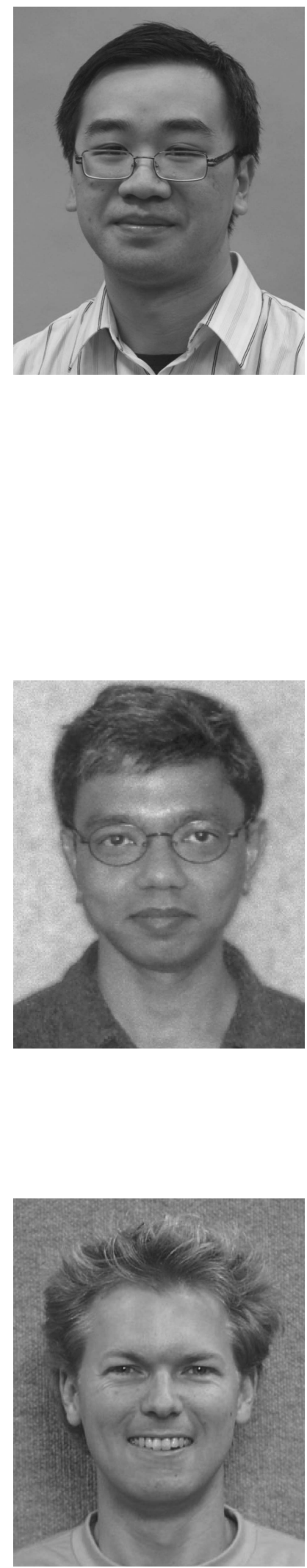

Alex Leong (M'08) was born in Macau in 1980. He received the B.S. degree in mathematics and B.E. degree in electrical engineering in 2003, and the Ph.D. degree in electrical engineering in 2008, all from the University of Melbourne, Parkville, Australia.

$\mathrm{He}$ is currently a research fellow in the Department of Electrical and Electronic Engineering, University of Melbourne. His research interests include statistical signal processing, signal processing for sensor networks, and networked control systems.

Dr. Leong was the recipient of the L. R. East Medal from Engineers Australia in 2003, and an Australian Postdoctoral Fellowship from the Australian Research Council in 2009.

Subhrakanti Dey (M'96-SM'06) was born in Calcutta, India, in 1968. He received the B.Tech. and M.Tech. degrees from the Department of Electronics and Electrical Communication Engineering, Indian Institute of Technology, Kharagpur, India in 1991 and 1993, respectively, and the Ph.D. degree from the Department of Systems Engineering, Research School of Information Sciences and Engineering, Australian National University, Canberra, Australia in 1996.

He has been with the Department of Electrical and Electronic Engineering, University of Melbourne, Parkville, Australia since February 2000, where he is currently a full professor. From September 1995 to September 1997 and September 1998 to February 2000, he was a postdoctoral research fellow with the Department of Systems Engineering, Australian National University. From September 1997 to September 1998, he was a post-doctoral research associate with the Institute for Systems Research, University of Maryland, College Park. His current research interests include networked control systems, wireless communications and networks, signal processing for sensor networks, and stochastic and adaptive estimation and control.

Dr. Dey currently serves on the Editorial Board of the IEEE Transactions on Signal Processing and Elsevier Systems and Control Letters. He was also an Associate Editor for the IEEE Transactions on Automatic Control during 2005-2007.

Jamie Evans (M'98) was born in Newcastle, Australia in 1970. He received the B.S. degree in physics and the B.E. degree in computer engineering from the University of Newcastle, in 1992 and 1993, respectively, where he received the University Medal upon graduation. He received the M.S. and the Ph.D. degrees from the University of Melbourne, Parkville, Australia, in 1996 and 1998, respectively, both in electrical engineering, and was awarded the Chancellor's Prize for excellence for his Ph.D. thesis.

From March 1998 to June 1999, he was a visiting researcher in the Department of Electrical Engineering and Computer Science, University of California, Berkeley. He returned to Australia to take up a position as lecturer at the University of Sydney, Australia, where he stayed until July 2001. Since that time, he has been with the Department of Electrical and Electronic Engineering, University of Melbourne, where he is now a full professor. His research interests are in communications theory, information theory, and statistical signal processing with a focus on wireless communications networks. 